\title{
Berry-Esseen theorem and local limit theorem for non uniformly expanding maps
}

\author{
Sébastien Gouëzel \\ Département de mathématiques et applications, École normale supérieure, 45, rue d'Ulm 75005 Paris, France
}

Received 28 June 2004; accepted 24 September 2004

Available online 29 January 2005

\begin{abstract}
In Young towers with sufficiently small tails, the Birkhoff sums of Hölder continuous functions satisfy a central limit theorem with speed $O(1 / \sqrt{n})$, and a local limit theorem. This implies the same results for many non uniformly expanding dynamical systems, namely those for which a tower with sufficiently fast returns can be constructed.

() 2005 Elsevier SAS. All rights reserved.

\section{Résumé}

Dans les tours de Young ayant des queues suffisamment petites, les sommes de Birkhoff des fonctions hölderiennes satisfont le théorème central limite avec vitesse $O(1 / \sqrt{n})$ et le théorème de la limite locale. Par conséquent, de nombreux systèmes dynamiques non uniformément dilatants satisfont les mêmes conclusions : il suffit de pouvoir construire une tour avec des retours à la base suffisamment rapides.
\end{abstract}

๑) 2005 Elsevier SAS. All rights reserved.

MSC: 37A30; 37A50; 37C30; 37E05; 47A56; 60F15

Keywords: Non uniformly expanding maps; Berry-Esseen theorem; Local limit theorem

\section{Results}

\subsection{Introduction}

Let $T: X \rightarrow X$ be a probability preserving transformation and $f: X \rightarrow \mathbb{R}$. The functions $f \circ T^{k}$, for $k \in \mathbb{N}$, are identically distributed random variables, and it is an important problem in ergodic theory to see whether they satisfy the same kind of limit theorems as independent random variables.

E-mail address: Sebastien.Gouezel@ens.fr (S. Gouëzel). 
Many results are known when $T$ is uniformly expanding or uniformly hyperbolic (without or with singularities, in the Markov or non Markov case), and $f$ is Hölder continuous. In this case, it is indeed often possible to construct a space of functions containing $f$ on which the transfer operator associated to $T$ has a spectral gap. Therefore, the spectral perturbation method, introduced by Nagaev in the case of Markov chains, makes it possible to mimic the probabilistic proofs on independent variables. In this way, it is possible to get distributional convergence (to normal laws or stable laws), and more subtle results such as the speed of convergence (also called the BerryEsseen theorem) or the local limit theorem (see for example [30,17,8,3]). These results, in turn, have important consequences concerning the asymptotic behavior of the system $[2,33]$.

On the other hand, when the system is not uniformly expanding or uniformly hyperbolic, it is not possible to use directly the aforementioned spectral method. Consequently, other methods have been devised to handle the distributional convergence of Birkhoff sums. Among many techniques, the most flexible one is probably the martingale argument of Gordin (see for example $[23,26,10,11,36])$. Some results have also been obtained on the speed in the central limit theorem, by direct estimates (see [22,27]). However, there is currently no result concerning the local limit theorem, which is not surprising since the proof of this theorem requires a heavy Fourier machinery, even in the probabilistic case, and is not easily accessible to elementary methods.

The aim of this article is to prove the local limit theorem and the Berry-Esseen theorem for Hölder functions in the setting of Young towers [35], where the decay of correlations is not exponential and the transfer operator has no spectral gap. The Young towers are abstract spaces which can be used to model many non uniformly expanding maps, for example the Pomeau-Manneville maps in dimension 1 studied by Liverani, Saussol and Vaienti [24], the Viana map (for which a tower is built in [5]), or the unimodal maps for which the critical point does not return too quickly close to itself [9]. Thus, all these maps also satisfy the local limit theorem, and the central limit theorem with speed $O(1 / \sqrt{n})$. These results also apply in non uniformly hyperbolic settings, with the techniques of [34].

The proof is spectral: it uses perturbations of transfer operators, as in [17], but applied to first return transfer operators associated to an induced map, as defined by Sarig in [32]. The method is related to [14], with a more systematic use of Banach algebra techniques.

\subsection{Results in Young towers}

A Young tower [35] is a probability space $(X, m)$ with a partition $\left(B_{i, j}\right)_{i \in I, j<\varphi_{i}}$ of $X$ by positive measure subsets, where $I$ is finite or countable and $\varphi_{i} \in \mathbb{N}^{*}$, together with a nonsingular map $T: X \rightarrow X$ satisfying the following properties.

1. $\forall i \in I, \forall 0 \leqslant j<\varphi_{i}-1, T$ is a measure preserving isomorphism between $B_{i, j}$ and $B_{i, j+1}$.

2. For every $i \in I, T$ is an isomorphism between $B_{i, \varphi_{i}-1}$ and $B:=\bigcup_{k \in I} B_{k, 0}$.

3. Let $\varphi$ be the function equal to $\varphi_{i}$ on $B_{i, 0}$, whence $T^{\varphi}$ is a function from $B$ to itself. Let $s(x, y)$ be the separation time of the points $x$ and $y \in B$ under $T^{\varphi}$, i.e. $s(x, y)=\inf \left\{n \mid \exists i \neq j,\left(T^{\varphi}\right)^{n}(x) \in B_{i, 0},\left(T^{\varphi}\right)^{n}(y) \in B_{j, 0}\right\}$.

As $T^{\varphi_{i}}$ is an isomorphism between $B_{i, 0}$ and $B$, it is possible to consider the inverse $g_{m}$ of its jacobian with respect to the measure $m$. We assume that there exist constants $\beta<1$ and $C>0$ such that $\forall x, y \in B_{i, 0}$, $\left|\log g_{m}(x)-\log g_{m}(y)\right| \leqslant C \beta^{s(x, y)}$.

4. The map $T$ preserves the measure $m$.

5. The partition $\bigvee_{0}^{\infty} T^{-n}\left(\left(B_{i, j}\right)\right)$ separates the points.

The notion of Young tower has been introduced by Young in [34,35] as a model for non uniformly expanding dynamical systems. The non uniformity is measured by the size of tails $m\{x \in B \mid \varphi(x)>n\}$ : if this quantity is very small, then most points enjoy some expansion before time $n$, when they first return to the basis. This expansion, in turn, is sufficient to study statistical properties of the system, including decay of correlations. Young has proved that, if $m[\varphi>n]=O\left(1 / n^{\beta}\right)$ for some $\beta>1$, then the correlations of sufficiently regular functions (see 
the definition of $C_{\tau}(X)$ below) decay like $O\left(1 / n^{\beta-1}\right)$. In particular, if $\beta>2$, these correlations are summable, and a martingale method can be used to prove that a central limit theorem holds.

We extend the separation time $s$ to the whole tower, by setting $s(x, y)=0$ if $x$ and $y$ are not in the same set $B_{i, j}$, and $s(x, y)=s\left(x^{\prime}, y^{\prime}\right)+1$ otherwise, where $x^{\prime}$ and $y^{\prime}$ are the next iterates of $x$ and $y$ in $B$. For $0<\tau<1$, set

$$
C_{\tau}(X)=\left\{f: X \rightarrow \mathbb{R}|\exists C>0, \forall x, y \in X,| f(x)-f(y) \mid \leqslant C \tau^{s(x, y)}\right\} .
$$

This space has a norm $\|f\|_{\tau}=\inf \left\{C|\forall x, y \in X| f,(x)-f(y) \mid \leqslant C \tau^{s(x, y)}\right\}+\|f\|_{\infty}$.

The following theorem is well known and can for example be proved using martingale techniques (see [35, Theorem 4]).

Theorem 1.1. Let $\tau<1$. Assume that $m[\varphi>n]=O\left(1 / n^{\beta}\right)$ with $\beta>2$. Let $f \in C_{\tau}(X)$ have a vanishing integral. Then there exists $\sigma^{2} \geqslant 0$ such that

$$
\frac{1}{\sqrt{n}} \sum_{k=0}^{n-1} f \circ T^{k} \rightarrow \mathcal{N}\left(0, \sigma^{2}\right)
$$

Moreover, $\sigma^{2}=0$ if and only if $f$ is a coboundary, i.e. there exists a measurable function $g$ such that $f=g-g \circ T$ almost everywhere.

The main results of this article are Theorems 1.2 and 1.3. To formulate the first one, we will need the following definition:

Definition. A map $f: X \rightarrow \mathbb{R}$ is periodic if there exist $\rho \in \mathbb{R}, g: X \rightarrow \mathbb{R}$ measurable, $\lambda>0$ and $q: X \rightarrow \mathbb{Z}$, such that $f=\rho+g-g \circ T+\lambda q$ almost everywhere. Otherwise, it is aperiodic.

Theorem 1.2 (local limit theorem). Let $\tau<1$. Assume that $m[\varphi>n]=O\left(1 / n^{\beta}\right)$ with $\beta>2$. Let $f \in C_{\tau}(X)$ have a vanishing integral, and let $\sigma^{2}$ be given by Theorem 1.1 .

Assume that $f$ is aperiodic. This implies in particular $\sigma^{2}>0$. Then, for any bounded interval $J \subset \mathbb{R}$, for any real sequence $k_{n}$ with $k_{n} / \sqrt{n} \rightarrow \kappa \in \mathbb{R}$, for any $u \in C_{\tau}(X)$, for any $v: X \rightarrow \mathbb{R}$ measurable,

$$
\sqrt{n} m\left\{x \in X \mid S_{n} f(x) \in J+k_{n}+u(x)+v\left(T^{n} x\right)\right\} \rightarrow|J| \frac{\mathrm{e}^{-\kappa^{2} /\left(2 \sigma^{2}\right)}}{\sigma \sqrt{2 \pi}} .
$$

The function on the right is the density of $\mathcal{N}\left(0, \sigma^{2}\right)$ : this theorem (for $u=v=0$ and $k_{n}=\kappa \sqrt{n}$ ) means that

$$
m\left\{\frac{1}{\sqrt{n}} S_{n} f \in \kappa+\frac{J}{\sqrt{n}}\right\} \sim P\left(\mathcal{N}\left(0, \sigma^{2}\right) \in \kappa+\frac{J}{\sqrt{n}}\right) .
$$

Hence, it shows that $S_{n} f / \sqrt{n}$ behaves like $\mathcal{N}\left(0, \sigma^{2}\right)$ at the local level (contrary to Theorem 1.1 which deals with the global level). It is important that $f$ is aperiodic. Otherwise, $f$ could be integer valued, and the theorem could not hold, e.g. for $k_{n}=0, u=v=0$ and $J=[1 / 3,2 / 3]$.

For $f: X \rightarrow \mathbb{R}$, define a function $f_{B}$ on $B$ by

$$
f_{B}(x)=\sum_{k=0}^{\varphi(x)-1} f\left(T^{k} x\right)
$$

In the probabilistic case, the Berry-Esseen theorem, giving the speed of convergence in the central limit theorem, holds under an $L^{3}$ moment condition [12]. In the dynamical setting, we will need the same kind of hypothesis, but on the function $f_{B}$. Note that, since $\left|f_{B}\right| \leqslant\|f\|_{\infty} \varphi$ and $\beta>2$, we always have $f_{B} \in L^{2}(B)$. 
Theorem 1.3 (speed in the central limit theorem). Let $\tau<1$. Assume that $m[\varphi>n]=O\left(1 / n^{\beta}\right)$ with $\beta>2$. Let $f \in C_{\tau}(X)$ have a vanishing integral, and $\sigma^{2}$ be given by Theorem 1.1 .

Assume that $\sigma^{2}>0$, and that there exists $0<\delta \leqslant 1$ such that $\int\left|f_{B}\right|^{2} 1_{\left|f_{B}\right|>z} \mathrm{~d} m=O\left(z^{-\delta}\right)$ when $z \rightarrow \infty$. If $\delta=1$, assume also that $\int f_{B}^{3} 1_{\left|f_{B}\right| \leqslant z} \mathrm{~d} m=O(1)$. Then there exists $C>0$ such that $\forall n \in \mathbb{N}^{*}, \forall a \in \mathbb{R}$,

$$
\left|m\left\{x \mid \frac{1}{\sqrt{n}} S_{n} f(x) \leqslant a\right\}-P\left(\mathcal{N}\left(0, \sigma^{2}\right) \leqslant a\right)\right| \leqslant \frac{C}{n^{\delta / 2}} .
$$

When $f_{B} \in L^{p}$ for some $2<p \leqslant 3$, then the conditions of the theorem are satisfied for $\delta=p-2$. In particular, when $f_{B} \in L^{3}$, we obtain a convergence with speed $O(1 / \sqrt{n})$, which is the usual Berry-Esseen theorem. Note also that, for any $f \in C_{\tau}(X)$, the conditions of the theorem are satisfied for $\delta=\beta-2$ if $2<\beta<3$, and for $\delta=1$ if $\beta>3$. The formulation we have given is more precise than the usual Berry-Esseen theorem, in view of the applications, where an $L^{p}$ condition would not be optimal (see for example Theorem 1.5). In fact, the conditions of the theorem on $f_{B}$ correspond to necessary and sufficient conditions to get a central limit theorem with speed $O\left(n^{-\delta / 2}\right)$ in the probabilistic (independent identically distributed) setting, as shown in [19, Theorem 3.4.1].

Remark. Using the same methods, it is possible to prove the same results in a more general setting, namely maps for which a first return map is Gibbs-Markov in the sense of [1]. For the sake of simplicity, we will only consider Young towers.

\subsection{Applications}

\subsubsection{General setting}

Let $(X, d)$ be a locally compact separable metric space, endowed with a Borel probability measure $\mu$, and $T: X \rightarrow X$ a nonsingular map for which $\mu$ is ergodic. Assume that there exist a bounded subset $B$ of $X$ with $\mu(B)>0$, a finite or countable partition $(\bmod 0)\left(B_{i}\right)_{i \in I}$ of $B$, with $\mu\left(B_{i}\right)>0$, and integers $\varphi_{i}>0$ such that:

1. $\forall i \in I, T^{\varphi_{i}}$ is an isomorphism between $B_{i}$ and $B$.

2. $\exists \lambda>1$ such that, $\forall i \in I, \forall x, y \in B_{i}, d\left(T^{\varphi_{i}} x, T^{\varphi_{i}} y\right) \geqslant \lambda d(x, y)$.

3. $\exists C>0$ such that, $\forall i \in I, \forall x, y \in B_{i}, \forall k<\varphi_{i}, d\left(T^{k} x, T^{k} y\right) \leqslant C d\left(T^{\varphi_{i}} x, T^{\varphi_{i}} y\right)$.

4. $\exists \theta>0$ and $D>0$ such that, $\forall i \in I$, the jacobian $g_{\mu}$ defined on $B_{i}$ by $g_{\mu}(x)=\mathrm{d} \mu / \mathrm{d}\left(\mu \circ T_{\mid B_{i}}^{\varphi_{i}}\right)$ satisfies: for all $x, y \in B_{i},\left|\log g_{\mu}(x)-\log g_{\mu}(y)\right| \leqslant D d\left(T^{\varphi_{i}} x, T^{\varphi_{i}} y\right)^{\theta}$.

Denote by $\varphi$ the function on $B$ equal to $\varphi_{i}$ on each $B_{i}$. If $\mu\{x \mid \varphi(x)>n\}$ is summable, we can define a space $X^{\prime}=\{(y, j) \mid y \in B, j<\varphi(x)\}$, and a map $T^{\prime}: X^{\prime} \rightarrow X^{\prime}$ by $T^{\prime}(y, j)=(y, j+1)$ if $j<\varphi(x)-1$ and $T^{\prime}(y, j)=\left(T^{\varphi(y)}(y), 0\right)$ otherwise. Define also $\pi: X^{\prime} \rightarrow X$ by $\pi(y, j)=T^{j}(y)$. Then $\pi \circ T^{\prime}=T \circ \pi$.

Set $\mu^{\prime}=\sum_{n=0}^{\infty} T_{*}^{\prime n}(\mu \mid B \cap\{\varphi>n\})$ : it is a measure of finite mass on $X^{\prime}$, not necessarily $T^{\prime}$-invariant. Young has proved in [35, Theorem 1] that there exists a unique invariant probability measure $m^{\prime}$ on $X^{\prime}$ which is absolutely continuous with respect to $\mu^{\prime}$. It is ergodic, and $\left(X^{\prime}, T^{\prime}, \mu^{\prime}\right)$ is a Young tower in the sense of Section 1.2. The measure $m=\pi_{*}\left(m^{\prime}\right)$ is $T$-invariant, absolutely continuous and ergodic.

If $f: X \rightarrow \mathbb{R}$ is Hölder continuous, then $f^{\prime}:=f \circ \pi: X^{\prime} \rightarrow \mathbb{R}$ belongs to $C_{\tau}\left(X^{\prime}\right)$ for $\tau$ close enough to 1 . Moreover, the Birkhoff sums $\sum_{k=0}^{n-1} f \circ T^{k}$ and $\sum_{k=0}^{n-1} f^{\prime} \circ T^{\prime k}$ have the same distribution with respect respectively to $m$ and $m^{\prime}$. Hence, Theorems 1.1, 1.2 and 1.3 on the function $f^{\prime}$ in the Young tower $\left(X^{\prime}, T^{\prime}, m^{\prime}\right)$ imply the same results on the function $f$ in $(X, T, m)$.

To apply these theorems, we have to check their assumptions. The condition $m[\varphi>n]=O\left(1 / n^{\beta}\right)$ with $\beta>2$ corresponds simply to the requirement

$$
\sum_{\varphi_{i}>n} \mu\left(B_{i}\right)=O\left(\frac{1}{n^{\beta}}\right) \text { for some } \beta>2 .
$$


To apply Theorem 1.2, we additionally have to check that the function $f^{\prime}$ is aperiodic for $T^{\prime}$, which can be complicated when the extension $X^{\prime}$ is not explicitly described. On the other hand, the aperiodicity of $f$ may be easier to check, using for example the information at the periodic points. In this case, the following abstract theorem ensures that $f^{\prime}$ is automatically aperiodic, whence we can apply Theorem 1.2.

Theorem 1.4. Let $T^{\prime}: X^{\prime} \rightarrow X^{\prime}$ be a probability preserving map on a probability space $\left(X^{\prime}, m^{\prime}\right)$. Let $(X, m)$ be a standard probability space, $T: X \rightarrow X$ an ergodic probability preserving map, and $\pi: X^{\prime} \rightarrow X$ a map with countable fibers, such that $m=\pi_{*}\left(m^{\prime}\right)$ and $T \circ \pi=\pi \circ T^{\prime}$. Let $f: X \rightarrow \mathbb{R}$. Then

- The function $f$ is a coboundary for $T$ if and only the function $f \circ \pi$ is a coboundary for $T^{\prime}$.

- The function $f$ is aperiodic for $T$ if and only if the function $f \circ \pi$ is aperiodic for $T^{\prime}$.

\subsubsection{Examples}

Recently, many maps have been shown to fit in the previous setting. For example, [5, Theorem 3] shows that the Alves-Viana map, given by

$$
T:\left\{\begin{array}{l}
S^{1} \times \mathbb{R} \rightarrow S^{1} \times \mathbb{R} \\
(\omega, x) \mapsto\left(16 \omega, a-x^{2}+\varepsilon \sin (2 \pi \omega)\right)
\end{array}\right.
$$

satisfies these assumptions (for any $\beta>2$ ) when 0 is preperiodic for the map $x \mapsto a-x^{2}$, and $\varepsilon$ is small enough. In fact, any map close enough to $T$ in the $C^{3}$-topology also satisfies them.

In the one-dimensional case, [9] shows that many unimodal maps of the interval also satisfy these hypotheses: it is sufficient that the returns of the critical point close to itself occur at a slow enough rate.

Finally, we will discuss with more details the case of the Pomeau-Manneville maps, studied among many others by Liverani, Saussol and Vaienti [24]. They form an interesting class of applications, since the influence of the fixed point 0 becomes more and more important when $\alpha$ increases. The explicit formula (2) is not important, what matters is only the local behavior around the fixed point. Hence, all the following results can be extended to a much larger class of examples but, for the sake of simplicity, we will only consider the following maps.

Let $\alpha \in(0,1 / 2)$, and consider $T:[0,1] \rightarrow[0,1]$ given by

$$
T(x)= \begin{cases}x\left(1+2^{\alpha} x^{\alpha}\right) & \text { if } 0 \leqslant x \leqslant 1 / 2, \\ 2 x-1 & \text { if } 1 / 2<x \leqslant 1\end{cases}
$$

This map has a parabolic fixed point at 0 , and is expanding elsewhere. It has a unique absolutely continuous invariant probability measure $m$, whose density is Lipschitz on any interval of the form $(\varepsilon, 1]$ [24, Lemma 2.3].

Theorem 1.5. Let $0<\alpha<1 / 2$, and let $f:[0,1] \rightarrow \mathbb{R}$ be a Hölder function with vanishing integral, which cannot be written as $g-g \circ T$. Then $f$ satisfies a central limit theorem with variance $\sigma^{2}>0$.

- If $\alpha<1 / 3$, or $f(0)=0$ and there exists $\gamma>\alpha-1 / 3$ such that $|f(x)| \leqslant K x^{\gamma}$, then there exists $C>0$ such that $\forall n \in \mathbb{N}^{*}, \forall a \in \mathbb{R}$,

$$
\left|m\left\{x \mid \frac{1}{\sqrt{n}} S_{n} f(x) \leqslant a\right\}-P\left(\mathcal{N}\left(0, \sigma^{2}\right) \leqslant a\right)\right| \leqslant \frac{C}{\sqrt{n}} .
$$

- If $1 / 3<\alpha<1 / 2, f(0)=0$ and there exists $\gamma>0$ such that $|f(x)| \leqslant K x^{\gamma}$ and $\delta:=\frac{1}{\alpha-\gamma}-2 \in(0,1)$, then there exists $C>0$ such that $\forall n \in \mathbb{N}^{*}, \forall a \in \mathbb{R}$,

$$
\left|m\left\{x \mid \frac{1}{\sqrt{n}} S_{n} f(x) \leqslant a\right\}-P\left(\mathcal{N}\left(0, \sigma^{2}\right) \leqslant a\right)\right| \leqslant \frac{C}{n^{\delta / 2}} .
$$


- If $1 / 3<\alpha<1 / 2$ and $f(0) \neq 0$, then there exists $C>0$ such that $\forall n \in \mathbb{N}^{*}, \forall a \in \mathbb{R}$,

$$
\left|m\left\{x \mid \frac{1}{\sqrt{n}} S_{n} f(x) \leqslant a\right\}-P\left(\mathcal{N}\left(0, \sigma^{2}\right) \leqslant a\right)\right| \leqslant \frac{C}{n^{(1 / 2 \alpha)-1}} .
$$

\section{Moreover, if $f$ is aperiodic, it satisfies the local limit theorem.}

Proof. Let $x_{0}=1$, and $x_{n+1}$ be the preimage of $x_{n}$ in [0,1/2]. Let $y_{n+1}$ be the preimage of $x_{n}$ in $(1 / 2,1]$ : the intervals $B_{n}=\left(y_{n+1}, y_{n}\right]$ form a partition of $B=(1 / 2,1]$ and, if $\varphi_{n}=n$, all the hypotheses of Section 1.3 are satisfied. Moreover, $m\left(B_{n}\right) \sim \frac{C}{n^{1 / \alpha+1}}$ and $x_{n} \sim \frac{D}{n^{1 / \alpha}}$ for constants $C, D>0$ [24]. In particular, $m(\varphi>n)=O\left(1 / n^{\beta}\right)$ for $\beta=1 / \alpha>2$.

Let $f$ be Hölder on $[0,1]$. If $f(0) \neq 0$, then $f_{B}=n f(0)+o(n)$ on $B_{n}$. Otherwise, let $\gamma>0$ be such that $|f(x)| \leqslant K x^{\gamma}$. Reducing $\gamma$ if necessary, we can assume that $\gamma<\alpha$. Then it is easy to check that $\left|f_{B}\right| \leqslant C n^{1-\gamma / \alpha}$ on $B_{n}$.

Using these estimates, we can check the integrability assumptions of Theorem 1.3 for $\delta=1$ in the first case, $\frac{1}{\alpha-\gamma}-2$ in the second case, and $\frac{1}{\alpha}-2$ in the third case. Hence, Theorem 1.3 implies the desired estimates on the speed in the central limit theorem.

Finally, the local limit theorem is a direct consequence of Theorem 1.2.

The aperiodicity assumption is a priori not easy to check, since the periodicity equality $f=g-g \circ T+\rho+\lambda q$ is assumed to hold only almost everywhere. However, under suitable regularity assumptions on $f$, it is possible to prove that this equality holds everywhere (see e.g. [3] for locally constant $f$, [15] for Hölder $f$ ). For example, if $T$ is given by (2), then $f=\log \left|T^{\prime}\right|-\int \log \left|T^{\prime}\right|$ is aperiodic.

In Section 2, we will prove Theorem 1.4, and show that it is sufficient to prove Theorems 1.2 and 1.3 in mixing Young towers (i.e., such that the return times $\varphi_{i}$ satisfy $\operatorname{gcd}\left(\varphi_{i}\right)=1$ ). The rest of paper is devoted to the proof of these theorems. In Section 3, we prove an abstract spectral result on perturbations of series of operators. In Section 4, we apply this result to first return transfer operators, to get the key result Theorem 4.6. We then use this estimate in the last two sections to prove respectively the local limit Theorem 1.2 and the Berry-Esseen Theorem 1.3.

\section{Preliminary reductions}

\subsection{Proof of Theorem 1.4}

Proof of the coboundary result. If $f$ is a coboundary, i.e. $f=g-g \circ T$, then $f^{\prime}:=f \circ \pi$ can be written as $f^{\prime}=g^{\prime}-g^{\prime} \circ T^{\prime}$, where $g^{\prime}=g \circ \pi$. However, the converse is not immediate: if $f^{\prime}=g^{\prime}-g^{\prime} \circ T^{\prime}$, the function $g^{\prime}$ is a priori not constant on the fibers $\pi^{-1}(x)$, which prevents us from writing $g^{\prime}=g \circ \pi$.

We use the following characterization of coboundaries: Let $T$ be an endomorphism of a probability space $(X, m)$. Then a measurable function $f$ on $X$ can be written as $g-g \circ T$ if and only if

$$
\forall \varepsilon>0, \exists C>0, \forall n \geqslant 1, \quad m\left\{x \in X|| S_{n} f(x) \mid \geqslant C\right\} \leqslant \varepsilon .
$$

This characterization, due to Schmidt, is proved for example in [4].

If $f^{\prime}$ is a coboundary, then (3) is satisfied by $f^{\prime}$ in $X^{\prime}$, whence it is also satisfied by $f$ in $X$ (since this condition only involves distributions). Thus, $f$ can be written as $g-g \circ T$.

Proof of the aperiodicity result. If $f$ is periodic on $X$, i.e. $f=\rho+g-g \circ T+\lambda q$ where $q$ is integer-valued, then $f \circ \pi=\rho+(g \circ \pi)-(g \circ \pi) \circ T^{\prime}+\lambda(q \circ \pi)$, i.e. $f \circ \pi$ is periodic. On the other hand, if $f \circ \pi=\rho^{\prime}+g^{\prime}-$ $g^{\prime} \circ T^{\prime}+\lambda q^{\prime}$, it is not necessarily possible to write directly $g^{\prime}=g \circ \pi$. The proof of the periodicity of $f$ will use 
ideas of [4]. We can assume for example that $\lambda=2 \pi$. Replacing $m^{\prime}$ by one of its ergodic components, we can also assume that $m^{\prime}$ is ergodic.

Since the projection $\pi$ has countable fibers, there exists a measurable subset $A$ of $X^{\prime}$ such that $\pi$ is an isomorphism between $A$ and $X$, and $m^{\prime}(A)>0$. Define a function $\tilde{g}$ on $X$ by $\tilde{g}(x)=g^{\prime}\left(x^{\prime}\right)$, where $x^{\prime}$ is the unique preimage of $x$ in $A$. Replacing $f$ by $f-\tilde{g}+\tilde{g} \circ T-\rho^{\prime}$, and $g^{\prime}$ by $g^{\prime}-\tilde{g} \circ \pi$, we can assume without loss of generality that $g^{\prime}=0$ on $A$ and $\rho^{\prime}=0$.

For $x \in X$, let $W_{n}(x)$ be the measure on $S^{1}$ given by

$$
W_{n}(x)=\frac{1}{n} \sum_{k=1}^{n} \delta\left(\mathrm{e}^{\mathrm{i} S_{k} f(x)}\right)
$$

where $\delta(y)$ is the Dirac mass at $y$. For $u \in C^{0}\left(S^{1}\right)$, it is possible by compactness to find a subsequence $n_{k}$ such that

$$
\int_{S^{1}} u \mathrm{~d} W_{n_{k}}(x) \rightarrow L(u)(x) \quad \text { weak } * \text { in } L^{\infty}(X) .
$$

It is possible to obtain this convergence for a dense countable set of functions in $C^{0}\left(S^{1}\right)$, by a diagonal argument. By passing to a further subsequence, it is also possible to guarantee that $\frac{1}{n} \sum_{k=1}^{n} \int_{S^{1}} u \mathrm{~d} W_{n_{k}}(x) \rightarrow L(u)(x)$ on a set $Y \subset X$ with $m(Y)=1$, by Komlos' Theorem [21]. By density, we get the same convergence for any $u \in C^{0}\left(S^{1}\right)$.

For $x \in Y$, the map $u \in C^{0}\left(S^{1}\right) \mapsto L(u)(x) \in \mathbb{R}$ is a nonnegative continuous linear functional sending 1 to 1 , thus given by a probability measure $P_{x}$. Moreover, these measures satisfy $P_{T x}(S)=P_{x}\left(\mathrm{e}^{\mathrm{i} f(x)} S\right)$ for any Borel subset $S$ of $S^{1}$, since $W_{n}(T x)(S)=W_{n}(x)\left(\mathrm{e}^{\mathrm{i} f(x)} S\right) \pm \frac{2}{n}$.

For some $\varepsilon>0$, we will prove that

$$
m\left\{x \mid P_{x}(\{1\}) \geqslant \varepsilon\right\} \geqslant \varepsilon .
$$

If $x^{\prime} \in A \cap T^{\prime-k}(A)$, then $\mathrm{e}^{\mathrm{i} S_{k} f \circ \pi\left(x^{\prime}\right)}=\mathrm{e}^{\mathrm{i}\left(g^{\prime}\left(x^{\prime}\right)-g^{\prime} \circ T^{\prime k}\left(x^{\prime}\right)\right)}=1$, i.e. $S_{k} f \circ \pi\left(x^{\prime}\right) \in 2 \pi \mathbb{Z}$. Hence,

$$
\begin{aligned}
\int_{X} W_{n}(x)(\{1\}) \mathrm{d} m(x) & =\frac{1}{n} \sum_{k=1}^{n} \int_{X} 1\left(S_{k} f(x) \in 2 \pi \mathbb{Z}\right) \mathrm{d} m(x) \geqslant \frac{1}{n} \sum_{k=1}^{n} \int_{X^{\prime}} 1\left(A \cap T^{\prime-k} A\right) \mathrm{d} m^{\prime}\left(x^{\prime}\right) \\
& =\int_{X^{\prime}} 1_{A} \cdot\left(\frac{1}{n} \sum_{k=1}^{n} 1_{A} \circ T^{\prime k}\right) \mathrm{d} m^{\prime}\left(x^{\prime}\right) \rightarrow m^{\prime}(A)^{2}>0
\end{aligned}
$$

by Birkhoff Theorem. Thus, for large enough $n, \int_{X} W_{n}(x)(\{1\}) \geqslant 3 \varepsilon>0$, whence $\int\left(\frac{1}{n} \sum_{k=1}^{n} W_{n_{k}}(x)\right)(\{1\}) \geqslant 2 \varepsilon$ for large enough $n$. Since $\left(\frac{1}{n} \sum_{k=1}^{n} W_{n_{k}}(x)\right)(\{1\}) \leqslant 1$, we get

$$
m\left\{x \mid\left(\frac{1}{n} \sum_{k=1}^{n} W_{n_{k}}(x)\right)(\{1\}) \geqslant \varepsilon\right\} \geqslant \varepsilon .
$$

Thus, the set $C=\left\{x \mid \lim \sup \left(\frac{1}{n} \sum_{k=1}^{n} W_{n_{k}}(x)\right)(\{1\}) \geqslant \varepsilon\right\}$ satisfies $m(C) \geqslant \varepsilon$. Finally, $P_{x}(\{1\}) \geqslant \varepsilon$ on $C$, and this proves (4).

Define a measure $\mu$ on $X \times S^{1}$, by $\mu(U \times V)=\int_{U} P_{x}(V) \mathrm{d} m(x)$. Then $\mu$ is invariant under the action of $T_{f}:(x, y) \mapsto\left(T(x), \mathrm{e}^{-\mathrm{i} f(x)} y\right)$, and [4] proves that, for almost every ergodic component $P$ of $\mu$, there exists a compact subgroup $H$ of $S^{1}$ and a map $\omega: X \rightarrow S^{1}$ such that, denoting by $m_{H}$ the Haar measure of $H$,

$$
\forall U \times V \subset X \times S^{1}, \quad P(U \times V)=\int_{U} m_{H}(\omega(x) V) \mathrm{d} m(x) .
$$

Moreover, in this case, it is possible to write $\mathrm{e}^{\mathrm{i} f(x)}=\frac{\omega(T x)}{\omega(x)} \psi(x)$, where $\psi$ takes its values in $H$. 
If, for all component $P$ of $\mu$, we had $H=S^{1}$, then $P=m \otimes$ Leb for all $P$, whence $\mu=m \otimes$ Leb. This is a contradiction, since $\mu(C \times\{1\})>0$, while $(m \otimes$ Leb $)(C \times\{1\})=0$. Thus, for some choice of $P, H=\mathbb{Z} / k \mathbb{Z}$, whence $\psi(x)^{k}=1$, and $\mathrm{e}^{\mathrm{i} k f(x)}=\omega(T x)^{k} / \omega(x)^{k}$. Thus, $f$ is periodic on $X$.

Remark. The proof only shows that the period of $f$ on $X$ divides the period of $f \circ \pi$ on $X^{\prime}$, not that they are equal. In fact, it is not hard to construct examples of Young towers where the two periods are different. Moreover, the result is not true without the assumption that the fibers are countable.

\subsection{Reduction to the mixing case}

In the proofs in Young towers, it is often useful to assume that the tower is mixing, i.e. $\operatorname{gcd}\left(\varphi_{i}\right)=1$. This restriction may seem technical, but it is important (for example, without it, there is no decay of correlations any more). For limit theorems, however, it is irrelevant: Theorems 1.1, 1.2 and 1.3 for mixing towers imply the same results for general towers.

Proof. We assume that Theorems 1.1, 1.2 and 1.3 are true in any mixing Young tower. Let $X$ be a non-mixing Young tower, with $N=\operatorname{gcd}\left(\varphi_{i}\right)>1$, and let $f \in C_{\tau}(X)$ be of vanishing integral. For $k=0, \ldots, N-1$, set $Z_{k}=\bigcup B_{i, j}$ for $j \equiv k \bmod N$. Then, for every $k,\left(Z_{k}, T^{N}\right)$ is a mixing Young tower, to which we can apply Theorems 1.1, 1.2 and 1.3.

On $Z_{k}$, we consider the function $f_{k}$ given by $\sum_{i=0}^{N-1} f\left(T^{i} x\right)$, i.e. $\left(S_{N} f\right)_{\mid Z_{k}}$. Then $\int_{Z_{k}} f_{k}=\int_{X} f=0$. Theorem 1.1 applied to $f_{k}$ on $\left(Z_{k}, T^{N}\right)$ gives a constant $\sigma_{k}$ such that, on $Z_{k}$,

$$
\frac{1}{\sqrt{s N}} S_{s N} f \rightarrow \mathcal{N}\left(0, \sigma_{k}^{2}\right)
$$

Writing an integer $n$ as $s N+r$ with $r<N$, we get that $\frac{1}{\sqrt{n}} S_{n} f \rightarrow \mathcal{N}\left(0, \sigma_{k}^{2}\right)$ on $Z_{k}$. Finally, if $x \in Z_{0}, S_{n} f(x)$ and $S_{n} f\left(T^{k} x\right)$ differ by at most $2 k\|f\|_{\infty}$. Thus, $\frac{1}{\sqrt{n}} S_{n} f-\frac{1}{\sqrt{n}} S_{n} f \circ T^{k}$ tends to 0 in probability on $Z_{0}$, which shows that $\sigma_{k}=\sigma_{0}$. Writing $\sigma$ for this common number, we get that $\frac{1}{\sqrt{n}} S_{n} f \rightarrow \mathcal{N}\left(0, \sigma^{2}\right)$ on $X$. Moreover, if $\sigma=0$, then $f_{0}$ is a coboundary for $T^{N}$, i.e. $f_{0}=g-g \circ T^{N}$ where $g: Z_{0} \rightarrow \mathbb{R}$ is measurable. We extend $g$ to the whole tower: if $(x, j) \in X$, with $j=s N+r$ and $r<N$, set $g(x, j)=g(x, s N)-\sum_{i=0}^{r-1} f(x, s N+i)$. It is then easy to check that $f=g-g \circ T$. This proves Theorem 1.1.

For the local limit theorem, let us assume that $f$ is aperiodic, and take $u, v$ and $k_{n}$ as in the assumptions of Theorem 1.2. We show that the functions $f_{k}$ are also aperiodic. Otherwise, for example, $f_{0}=\rho+g-g \circ T^{N}+\lambda q$, where $g$ and $q$ are defined on $Z_{0}$. We extend $g$ and $q$ to the whole tower: for $(x, j) \in X$ with $j=s N+r$ and $0<r<$ $N$, set $g(x, j)=g(x, s N)-\sum_{0}^{r-1}\left(f(x, s N+i)-\frac{\rho}{N}\right)+\lambda q(x, s N)$, and $q(x, j)=0$. Then $f=\frac{\rho}{N}+g-g \circ T+\lambda q$, which is a contradiction. Thus, all the functions $f_{k}$ are aperiodic. We can apply to them Theorem 1.2 in the mixing tower $\left(Z_{k}, T^{N}\right)$, and get that

$$
\sqrt{s N} m\left\{x \in Z_{k} \mid S_{s N} f(x) \in J+k_{s N}+u(x)+v\left(T^{s N} x\right)\right\} \rightarrow m\left(Z_{k}\right)|J| \frac{\mathrm{e}^{-\kappa^{2} /\left(2 \sigma^{2}\right)}}{\sigma \sqrt{2 \pi}} .
$$

Summing over $k$, we get the conclusion of Theorem 1.2 for $f$, and for the times of the form $s N$. For times of the form $s N+r$ with $0<r<N$, we use the same result for $f \circ T^{r}, u-S_{r} f, v \circ T^{r}$ and the sequence $k_{s N+r}$, and get that

$$
\sqrt{s N+r} m\left\{x \in X \mid S_{s N} f \circ T^{r}(x) \in J+k_{s N+r}+u(x)-S_{r} f(x)+v\left(T^{s N+r} x\right)\right\} \rightarrow|J| \frac{\mathrm{e}^{-\kappa^{2} /\left(2 \sigma^{2}\right)}}{\sigma \sqrt{2 \pi}} .
$$

As $S_{S N} f \circ T^{r}(x)+S_{r} f(x)=S_{S N+r} f(x)$, this concludes the proof of Theorem 1.2. 
Finally, the central limit theorem with speed is deduced from the same result on each $Z_{k}$, for times of the form $s N$. We extend the result to arbitrary times: writing $n$ as $s N+r$ with $r<N$, we have $\left|S_{s N+r} f-S_{s N} f\right| \leqslant r\|f\|_{\infty}$. This introduces an error, of order $O(1 / \sqrt{n}) \leqslant O\left(1 / n^{\delta / 2}\right)$.

Remark. For this proof, it was important to have a strong version of the local limit theorem, involving functions $u$ and $v$.

Theorem 1.1 is proved in [35, Theorem 4]. The rest of the paper is devoted to the proof of Theorems 1.2 and 1.3 in mixing Young towers. From this point on, $X$ will be a mixing Young tower, i.e. $\operatorname{gcd}\left(\varphi_{i}\right)=1$.

\section{An abstract result}

If $\left(a_{n}\right)_{n \in \mathbb{N}}$ and $\left(b_{n}\right)_{n \in \mathbb{N}}$ are two sequences indexed by $\mathbb{N}$, we denote by $\left(a_{n}\right) \star\left(b_{n}\right)$ the sequence $c_{n}=$ $\sum_{k=0}^{n} a_{k} b_{n-k}$. If $\left(a_{n}\right)_{n \in \mathbb{Z}}$ and $\left(b_{n}\right)_{n \in \mathbb{Z}}$ are two summable sequences indexed by $\mathbb{Z}$, we also define their convolution $c_{n}=\left(a_{n}\right) \star\left(b_{n}\right)$ by $c_{n}=\sum_{k=-\infty}^{\infty} a_{k} b_{n-k}$. Hence, if $\sum a_{n} z^{n}$ and $\sum b_{n} z^{n}$ are series with summable coefficients, the coefficient of $z^{n}$ in $\left(\sum a_{k} z^{k}\right)\left(\sum b_{k} z^{k}\right)$ is given by $\left(a_{n}\right) \star\left(b_{n}\right)$ (more precisely, it is given by the $n$th term $\left(\left(a_{k}\right) \star\left(b_{k}\right)\right)_{n}$ of this sequence, but we will often abuse notations and write simply $\left.\left(a_{n}\right) \star\left(b_{n}\right)\right)$. Finally, we write $\mathbb{D}=\{z \in \mathbb{C}|| z \mid<1\}$ and $\overline{\mathbb{D}}=\{z \in \mathbb{C}|| z \mid \leqslant 1\}$.

The goal of this section is to prove the following theorem:

Theorem 3.1. Let $\beta>2$. Let $R_{n}$, for $n \in \mathbb{N}^{*}$, be operators on a Banach space $E$, with $\sum_{k=n+1}^{\infty}\left\|R_{k}\right\|=O\left(1 / n^{\beta}\right)$. Set $R(z)=\sum R_{n} z^{n}$, and assume that 1 is a simple isolated eigenvalue of $R(1)$, while $I-R(z)$ is invertible for $z \in \overline{\mathbb{D}}-\{1\}$. Let $P$ be the spectral projection associated to $R(1)$ and the eigenvalue 1 , and assume that $P R^{\prime}(1) P=$ $\mu P$ with $\mu>0$.

Let $R_{n}(t)$ be operators on $E$ (for $t$ in some interval $[-\alpha, \alpha]$ with $\alpha>0$ ) such that $\sum_{k=n+1}^{\infty}\left\|R_{k}(t)-R_{k}\right\| \leqslant$ $C|t| / n^{\beta-1}$. Set $R(z, t)=\sum z^{n} R_{n}(t)$. Let $\lambda(z, t)$ be the eigenvalue close to 1 of $R(z, t)$, for $(z, t)$ close to $(1,0)$. We assume that $\lambda(1, t)=1-M(t)$ with $M(t) \sim c t^{2}$ for some constant $c$ with $\operatorname{Re}(c)>0$.

Then, for small enough $t, I-R(z, t)$ is invertible for all $z \in \mathbb{D}$. Let us denote its inverse by $\sum T_{n}(t) z^{n}$. Then there exist $\alpha^{\prime}>0, d>0$ and $C>0$ such that, for every $t \in\left[-\alpha^{\prime}, \alpha^{\prime}\right]$, for every $n \in \mathbb{N}^{*}$,

$$
\left\|T_{n}(t)-\frac{1}{\mu}\left(1-\frac{1}{\mu} M(t)\right)^{n} P\right\| \leqslant \frac{C}{n^{\beta-1}}+C|t|\left(\frac{1}{n^{\beta-1}}\right) \star\left(1-d t^{2}\right)^{n} .
$$

In the application of Theorem 3.1 to the proof of Theorems 1.2 and 1.3, the operators $R_{n}$ will describe the returns to the basis $B$, and will be easily understood, as well as their perturbations $R_{n}(t)$. On the other hand, $T_{n}$ will describe all the iterates at time $n$, and $T_{n}(t)$ will be closely related to the characteristic function $E\left(\mathrm{e}^{\mathrm{i} t} S_{n} f\right)$. Thus, (5) will enable us to describe precisely $E\left(\mathrm{e}^{\mathrm{i} t S_{n} f}\right)$, and this information will be sufficient to get Theorems 1.2 and 1.3.

\subsection{Banach algebras and Wiener Lemma}

In this paragraph, we define some Banach algebras which will be useful in the following estimates. We postpone the proofs of the properties of these algebras to the Appendix.

A Banach algebra $\mathcal{A}$ is a complex Banach space with an associative multiplication $\mathcal{A} \times \mathcal{A} \rightarrow \mathcal{A}$ such that $\|A B\| \leqslant\|A\|\|B\|$, and a neutral element. The set of invertible elements is then an open subset of $\mathcal{A}$, on which the inversion is continuous.

Let $\mathcal{C}$ be a Banach algebra. If $\gamma>1$, we write $\mathcal{O}_{\gamma}(\mathcal{C})$ for the set of formal series $\sum_{n=-\infty}^{\infty} A_{n} z^{n}$, where $A_{n} \in \mathcal{C}$ and $\left\|A_{n}\right\|=O\left(1 /|n|^{\gamma}\right)$ when $n \rightarrow \pm \infty$, endowed with the standard product of power series, corresponding to the 
convolution of the sequences $\left(A_{n}\right)$ and $\left(B_{n}\right)$. It admits the naive norm $\sup _{n \in \mathbb{Z}}(|n|+1)^{\gamma}\left\|A_{n}\right\|$, for which it is not a Banach algebra. However, there exists a norm, equivalent to the previous one, which makes $\mathcal{O}_{\gamma}(\mathcal{C})$ into a Banach algebra (Proposition A.1). Moreover, this algebra satisfies a Wiener Lemma: if $A(z)=\sum A_{n} z^{n} \in \mathcal{O}_{\gamma}(\mathcal{C})$ is such that $A(z)$ is invertible for every $z \in S^{1}$, then $A$ is invertible in $\mathcal{O}_{\gamma}(\mathcal{C})$ (Theorem A.3).

We will also use the Banach algebra $\mathcal{O}_{\gamma}^{+}(\mathcal{C})$, given by the set of series $\sum A_{n} z^{n} \in \mathcal{O}_{\gamma}(\mathcal{C})$ such that $A_{n}=0$ for $n<0$. It is a closed subalgebra of $\mathcal{O}_{\gamma}(\mathcal{C})$, and it satisfies also a Wiener Lemma (Theorem A.4).

Notation. If $f:[-\alpha, \alpha] \times \mathbb{Z} \rightarrow \mathbb{R}_{+}$for some $\alpha>0$, and $\mathcal{C}$ is a Banach algebra, we denote by $O_{\mathcal{C}}(f(t, n))$ the set of series $\sum_{n=-\infty}^{\infty} c_{n}(t) z^{n}$ where $c_{n}:[-\alpha, \alpha] \rightarrow \mathcal{C}$ is such that there exists $\alpha^{\prime}>0$ and $C>0$ such that

$$
\forall t \in\left[-\alpha^{\prime}, \alpha^{\prime}\right], \forall n \in \mathbb{Z}, \quad\left\|c_{n}(t)\right\| \leqslant C f(t, n) .
$$

We will often omit the subscript in $O_{\mathcal{C}}$. As usual, we will often write $\sum c_{n}(t) z^{n}=O(f(t, n))$ instead of the more correct formulation $\sum c_{n}(t) z^{n} \in O(f(t, n))$. We will also write $O(g(n))$ for the set of series $\sum A_{n} z^{n}$ with $\left\|A_{n}\right\| \leqslant C g(n)$ for some constant $C$. This is a particular case of the previous notation, where the functions $f(t, n)$ are independent of $t$. Until the end of Section 3, the notation $O$ will always have this signification.

Remark. The notations $\mathcal{O}$ and $O$ should not be confused: there are similarities (which is why we have used the same letter), but the calligraphic notation $\mathcal{O}$ indicates additionally a Banach algebra. In this case, we can for example use the continuity of inversion.

With these notations, we can reformulate Theorem A.3 as follows: if $\sum A_{n} z^{n}=O\left(1 /(|n|+1)^{\gamma}\right)$ for $\gamma>1$, and $\sum A_{n} z^{n}$ is invertible for every $z \in S^{1}$, then $\left(\sum A_{n} z^{n}\right)^{-1}=O\left(1 /(|n|+1)^{\gamma}\right)$. The fact that $\mathcal{O}_{\gamma}(\mathcal{C})$ is a Banach algebra also implies that, for $\gamma>1$,

$$
O\left(\frac{1}{(|n|+1)^{\gamma}}\right) \star O\left(\frac{1}{(|n|+1)^{\gamma}}\right) \subset O\left(\frac{1}{(|n|+1)^{\gamma}}\right),
$$

i.e., if two series $\sum A_{n} z^{n}$ and $\sum B_{n} z^{n}$ (with $A_{n}, B_{n} \in \mathcal{C}$ ) satisfy

$$
\sup _{n \in \mathbb{Z}}(|n|+1)^{\gamma}\left\|A_{n}\right\|<\infty \text { and } \sup _{n \in \mathbb{Z}}(|n|+1)^{\gamma}\left\|B_{n}\right\|<\infty,
$$

then the series $\sum C_{n} z^{n}:=\left(\sum A_{n} z^{n}\right)\left(\sum B_{n} z^{n}\right)$ also satisfies $\sup _{n \in \mathbb{Z}}(|n|+1)^{\gamma}\left\|C_{n}\right\|<\infty$.

\subsection{Preliminary technical estimates}

For notational convenience, we will often write $t$ instead of $|t|$ in what follows. Equivalently, the reader may consider that the proofs are written for $t \geqslant 0$. We will also write $1 /|n|^{\gamma}$ instead of $1 /(|n|+1)^{\gamma}$, discarding the problem at $n=0$.

Lemma 3.2. When $\gamma>1$ and $d>0$,

$$
O\left(\frac{1}{|n|^{\gamma}}\right) \star O\left(1_{n \geqslant 0} t^{2}\left(1-d t^{2}\right)^{n}\right) \subset O\left(\frac{1}{|n|^{\gamma}}+1_{n \geqslant 0} t^{2}\left(1-\frac{d}{2} t^{2}\right)^{n}\right) .
$$

Proof. For $n<0$, the coefficient in the convolution is less than $\sum_{k=0}^{\infty} t^{2}\left(1-d t^{2}\right)^{k} \frac{1}{|n|^{\gamma}} \leqslant \frac{C}{|n|^{\gamma}}$. For $n \geqslant 0$, it is less than

$$
\sum_{k=-\infty}^{n / 2} \frac{1}{|k|^{\gamma}} t^{2}\left(1-d t^{2}\right)^{n / 2}+\sum_{k=n / 2}^{n} \frac{1}{(n / 2)^{\gamma}} t^{2}\left(1-d t^{2}\right)^{n-k} \leqslant C t^{2}\left(1-d t^{2}\right)^{n / 2}+\frac{C}{n^{\gamma}} .
$$

Finally, as $\sqrt{1-d t^{2}} \leqslant\left(1-\frac{d}{2} t^{2}\right)$, we get the conclusion. 
Lemma 3.3. Let $\gamma>1$ and $d>0$. Let $G_{t}(z)=O\left(t /|n|^{\gamma}+1_{n \geqslant 0} t^{3}\left(1-d t^{2}\right)^{n}\right)$, and assume that $F(z)=O\left(1 /|n|^{\gamma}\right)$ is invertible for every $z \in S^{1}$. Then $\left[F(z)+G_{t}(z)\right]^{-1}=F(z)^{-1}+O\left(\frac{t}{|n|^{\gamma}}+1_{n \geqslant 0} t^{3}\left(1-\frac{d}{64} t^{2}\right)^{n}\right)$.

Proof. We first assume that $F(z)=1$. Setting $H_{t}(z)=\sum_{n \in \mathbb{Z}} \frac{t}{|n|^{\gamma}} z^{n}+\sum_{n \in \mathbb{N}} t^{3}\left(1-d t^{2}\right)^{n} z^{n}$, the norm of the coefficients of $\left[1+G_{t}(z)\right]^{-1}$ is less than the coefficients of $\left[1-H_{t}(z)\right]^{-1}$. Thus, it is sufficient to consider $1 /\left(1-t K(z)-t^{3} /\left(1-\left(1-d t^{2}\right) z\right)\right)$ where $K(z)=\sum z^{n} /|n|^{\gamma}$. Note that

$$
\frac{1}{1-t K(z)-t^{3} /\left(1-\left(1-d t^{2}\right) z\right)}=\frac{1}{1-t K(z)-t^{3}} \frac{1-\left(1-d t^{2}\right) z}{1-\left(1-d t^{2}\right)\left[1+t^{3} /\left(1-t K(z)-t^{3}\right)\right] z} .
$$

For small enough $t,\left|\left(1-d t^{2}\right)\left[1+t^{3} /\left(1-t K(z)-t^{3}\right)\right]\right|<1$, whence

$$
\begin{aligned}
& \frac{1-\left(1-d t^{2}\right) z}{1-\left(1-d t^{2}\right)\left[1+t^{3} /\left(1-t K(z)-t^{3}\right)\right] z} \\
& =\left(1-\left(1-d t^{2}\right) z\right) \sum_{n=0}^{\infty}\left(1-d t^{2}\right)^{n}\left[1+\frac{t^{3}}{1-t K(z)-t^{3}}\right]^{n} z^{n} \\
& =1+\frac{t z\left(1-d t^{2}\right)}{1-t K(z)-t^{3}} t^{2} \sum_{n=0}^{\infty}\left(1-d t^{2}\right)^{n}\left[1+\frac{t^{3}}{1-t K(z)-t^{3}}\right]^{n} z^{n}
\end{aligned}
$$

We first study the sum. Let $\mathcal{A}=\mathcal{O}_{\gamma}(\mathbb{C})$ be the Banach algebra of the series whose coefficients are $O\left(1 /|n|^{\gamma}\right)$. As $K(z) \in \mathcal{A}$, we have $1-t K(z)-t^{3}=1+O_{\mathcal{A}}(t)$. Since the inversion is Lipschitz on a Banach algebra, we get $t^{3} /\left(1-t K(z)-t^{3}\right)=t^{3}+O_{\mathcal{A}}\left(t^{4}\right)$, whence $\left\|\left[1+t^{3} /\left(1-t K(z)-t^{3}\right)\right]^{n}\right\|_{\mathcal{A}} \leqslant C\left(1+2 t^{3}\right)^{n}$. Let us estimate the coefficient of $z^{p}$ in $t^{2} \sum_{n=0}^{\infty}\left(1-d t^{2}\right)^{n}\left[1+t^{3} /\left(1-t K(z)-t^{3}\right)\right]^{n} z^{n}$. This is at most

$$
\begin{aligned}
t^{2} \sum_{n=0}^{\infty}\left(1-d t^{2}\right)^{n}\left(\left[1+\frac{t^{3}}{1-t K(z)-t^{3}}\right]^{n}\right)_{-n+p} & \leqslant \sum_{n=0}^{\infty} t^{2}\left(1-d t^{2}\right)^{n} \frac{\left(1+2 t^{3}\right)^{n}}{|-n+p|^{\gamma}} \\
& \leqslant \sum_{n=0}^{\infty} t^{2}\left(1-\frac{d}{2} t^{2}\right)^{n} \frac{1}{|-n+p|^{\gamma}}
\end{aligned}
$$

for $t$ small enough so that $\left(1-d t^{2}\right)\left(1+2 t^{3}\right) \leqslant\left(1-\frac{d}{2} t^{2}\right)$. We find the same expression as in the convolution between $O\left(1_{n \geqslant 0} t^{2}\left(1-\frac{d}{2} t^{2}\right)^{n}\right)$ and $O\left(1 /|n|^{\gamma}\right)$, that we have already estimated in Lemma 3.2. Thus, we get at most $O\left(1 /|p|^{\gamma}+1_{p \geqslant 0} t^{2}\left(1-\frac{d}{4} t^{2}\right)^{p}\right)$.

As $t z\left(1-d t^{2}\right) /\left(1-t K(z)-t^{3}\right)=O\left(t /|n|^{\gamma}\right)$, another convolution yields that $(8)$ is

$$
1+O\left(\frac{t}{|n|^{\gamma}}+1_{n \geqslant 0} t^{3}\left(1-\frac{d}{8} t^{2}\right)^{n}\right)
$$

Multiplying by $1 /\left(1-t K(z)-t^{3}\right)=1+O\left(t /|n|^{\gamma}\right)$ gives that $(7)=1+O\left(t /|n|^{\gamma}+1_{n \geqslant 0} t^{3}\left(1-\frac{d}{16} t^{2}\right)^{n}\right)$. This concludes the proof in the case $F(z)=1$.

We now handle the case of an arbitrary $F(z)$. Note that

$$
\left[F(z)+G_{t}(z)\right]^{-1}=\left[1+F(z)^{-1} G_{t}(z)\right]^{-1} F(z)^{-1} .
$$

The Wiener Lemma A.3 implies that $F(z)^{-1}=O\left(1 /|n|^{\gamma}\right)$, whence Lemma 3.2 gives $F(z)^{-1} G_{t}(z)=$ $O\left(t /|n|^{\gamma}+1_{n \geqslant 0} t^{3}\left(1-\frac{d}{2} t^{2}\right)^{n}\right)$. Thus, the case $F(z)=1$ yields

$$
\left[1+F(z)^{-1} G_{t}(z)\right]^{-1}=1+O\left(\frac{t}{|n|^{\gamma}}+1_{n \geqslant 0} t^{3}\left(1-\frac{d}{32} t^{2}\right)^{n}\right) .
$$

Another convolution with $F(z)^{-1}$ gives the result. 


\subsection{Proof of Theorem 3.1}

Let $M(t)$ be as in Theorem 3.1. We fix once and for all $d>0$ such that $\left|1-\frac{1}{\mu} M(t)\right| \leqslant 1-d t^{2}$ for small enough $t$, and we restrict the range of $t$ so that this inequality is true. The invertibility of $R(z, t)$ for $z \in \mathbb{D}$ and small enough $t$ is proved in [14, Proposition 2.7].

To estimate the eigenvalues using Banach algebra techniques, we will need that the eigenvalue close to 1 of $R(z)$ is defined on the whole circle $S^{1}$, which is not a priori the case. Consequently, we use the construction in the second step of the proof of Theorem 2.4 in [14]: we replace $R(z)$ by $\tilde{R}(z)=\sum_{-\infty}^{\infty} \tilde{R}_{n} z^{n}$, such that it has a unique eigenvalue $\tilde{\lambda}(z)$ close to 1 for $z \in S^{1}$, equal to 1 only for $z=1$, with $\sum_{|k|>n}\left\|\tilde{R}_{k}\right\|=O\left(1 / n^{\beta}\right)$, and such that $R(z)=\tilde{R}(z)$ for $z$ close to 1 on $S^{1}$. We also set $\tilde{R}(z, t)=(\tilde{R}(z)-R(z))+\sum R_{n}(t) z^{n}$. For small enough $t$, $\tilde{R}(z, t)$ has for all $z \in S^{1}$ a unique eigenvalue $\tilde{\lambda}(z, t)$ close to 1 .

Lemma 3.4. We have

$$
\frac{1-\tilde{\lambda}(z, t)}{1-(1-(1 / \mu) M(t)) z}=\frac{1-\tilde{\lambda}(z)}{1-z}+O\left(\frac{t}{|n|^{\beta-1}}+1_{n \geqslant 0} t^{3}\left(1-\frac{d}{2} t^{2}\right)^{n}\right) .
$$

Proof. We write $K(z, t)=(\tilde{\lambda}(1, t)-\tilde{\lambda}(z, t)) /(1-z)-(1-\tilde{\lambda}(z)) /(1-z)$. Recall that $\tilde{\lambda}(1, t)=\lambda(1, t)=1-$ $M(t)$. Then, writing $B(z)=(1-\tilde{\lambda}(z)) /(1-z)$, we have

$$
\tilde{\lambda}(z, t)=1-M(t)+(z-1)(K(z, t)+B(z)) .
$$

Thus,

$$
\begin{aligned}
\frac{1-\tilde{\lambda}(z, t)}{1-(1-(1 / \mu) M(t)) z}-B(z) & =\frac{M(t)+(1-z)(K(z, t)+B(z))-[1-(1-(1 / \mu) M(t)) z] B(z)}{1-(1-(1 / \mu) M(t)) z} \\
& =K(z, t) \frac{1-z}{1-(1-(1 / \mu) M(t)) z}+M(t) \frac{1-z B(z) / \mu}{1-(1-(1 / \mu) M(t)) z} \\
& =\mathrm{I}+\mathrm{II} .
\end{aligned}
$$

For I,

$$
\frac{1-z}{1-(1-(1 / \mu) M(t)) z}=1-\frac{1}{\mu} M(t) \sum_{n=1}^{\infty}\left(1-\frac{1}{\mu} M(t)\right)^{n-1} z^{n}
$$

is in $1+O\left(1_{n \geqslant 0} t^{2}\left(1-d t^{2}\right)^{n}\right)$. We multiply it by $K(z, t)$. Set $\mathcal{A}=\mathcal{O}_{\beta-1}(\operatorname{Hom}(E))$ (the Banach algebra of functions whose coefficients are in $O\left(1 /|n|^{\beta-1}\right)$ for $\left.n \in \mathbb{Z}\right)$. We have $(\tilde{R}(z, t)-\tilde{R}(1, t)) /(z-1)=$ $(\tilde{R}(z)-\tilde{R}(1)) /(z-1)+O_{\mathcal{A}}(t)$. The proof of [14, Lemma 2.6], but in the algebra $\mathcal{A}$ and with tildes everywhere, applies (using Theorem A.3 to ensure that the inverses remain in $\mathcal{A})$. It gives $(\tilde{\lambda}(z, t)-\tilde{\lambda}(1, t)) /(z-1)=$ $(\tilde{\lambda}(z)-\tilde{\lambda}(1)) /(z-1)+O_{\mathcal{A}}(t)$, i.e. $K(z, t)=O\left(t /|n|^{\beta-1}\right)$. Hence, Lemma 3.2 yields

$$
I=O\left(\frac{t}{|n|^{\beta-1}}+1_{n \geqslant 0} t^{3}\left(1-\frac{d}{2} t^{2}\right)^{n}\right) .
$$

Since $(\tilde{R}(z)-\tilde{R}(1)) /(z-1)=O\left(1 /|n|^{\beta}\right)$, we prove that $B(z)=O\left(1 /|n|^{\beta}\right)$ as in the third step of the proof of Theorem 2.4 in [14] (but in the Banach algebra $\mathcal{O}_{\beta}(\operatorname{Hom}(E))$ ). Since $1-z B(z) / \mu$ vanishes at 1 (Step 7 of the proof of Lemma 3.1 in [16]) and is in $O\left(1 /|n|^{\beta}\right)$, it can be written as $(1-z) C(z)$ where $C(z)=O\left(1 /|n|^{\beta-1}\right)$. To obtain II, we multiply $C(z)$ by $M(t)(1-z) /(1-(1-(1 / \mu) M(t)) z)=O\left(t^{2}+1_{n \geqslant 0} t^{4}\left(1-d t^{2}\right)^{n}\right)$. Lemma 3.2 yields

$$
\mathrm{II}=O\left(\frac{t^{2}}{|n|^{\beta-1}}+1_{n \geqslant 0} t^{4}\left(1-\frac{d}{2} t^{2}\right)^{n}\right) .
$$


Corollary 3.5. We have

$$
\left(\frac{I-\tilde{R}(z, t)}{1-(1-(1 / \mu) M(t)) z}\right)^{-1}=\left(\frac{I-\tilde{R}(z)}{1-z}\right)^{-1}+O\left(\frac{t}{|n|^{\beta-1}}+1_{n \geqslant 0} t^{3}\left(1-\frac{d}{256} t^{2}\right)^{n}\right) .
$$

Proof. Let $\tilde{P}(z, t)$ be the spectral projection associated to the eigenvalue $\tilde{\lambda}(z, t)$ of $\tilde{R}(z, t)$, and $\tilde{Q}(z, t)=$ $I-\tilde{P}(z, t)$. Set $\mathcal{A}=\mathcal{O}_{\beta-1}(\operatorname{Hom}(E))$. Then $\tilde{R}(z, t)=\tilde{R}(z)+O_{\mathcal{A}}(t)$ by assumption. As $\mathcal{A}$ satisfies the Wiener Lemma A.3, the integral expression of the projection $\tilde{P}(z, t)$ shows that $\tilde{P}(z, t)=\tilde{P}(z)+O_{\mathcal{A}}(t)$. Moreover, $I-\tilde{R}(z, t) \tilde{Q}(z, t)=I-\tilde{R}(z) \tilde{Q}(z)+O_{\mathcal{A}}(t)$, whence $(I-\tilde{R}(z, t) \tilde{Q}(z, t))^{-1}=(I-\tilde{R}(z) \tilde{Q}(z))^{-1}+O_{\mathcal{A}}(t)$, since $I-\tilde{R}(z) \tilde{Q}(z)$ is invertible in $\mathcal{A}$ by Theorem A.3, and the inversion is Lipschitz.

As

$$
I-\tilde{R}(z, t)=(1-\tilde{\lambda}(z, t)) \tilde{P}(z, t)+(I-\tilde{R}(z, t) \tilde{Q}(z, t)) \tilde{Q}(z, t),
$$

Lemmas 3.3 and 3.4 yield that

$$
\begin{aligned}
( & \left.\frac{I-\tilde{R}(z, t)}{1-(1-(1 / \mu) M(t)) z}\right)^{-1} \\
= & \frac{1-(1-(1 / \mu) M(t)) z}{1-\tilde{\lambda}(z, t)} \tilde{P}(z, t)+\left(1-\left(1-\frac{1}{\mu} M(t)\right) z\right)(I-\tilde{R}(z, t) \tilde{Q}(z, t))^{-1} \tilde{Q}(z, t) \\
= & {\left[\frac{1-z}{1-\tilde{\lambda}(z)}+O\left(\frac{t}{|n|^{\beta-1}}+1_{n \geqslant 0} t^{3}\left(1-\frac{d}{128} t^{2}\right)^{n}\right)\right]\left[\tilde{P}(z)+O\left(\frac{t}{|n|^{\beta-1}}\right)\right] } \\
& +\left[(1-z)+O\left(1_{n=0} t^{2}\right)\right]\left[(I-\tilde{R}(z) \tilde{Q}(z))^{-1}+O\left(\frac{t}{|n|^{\beta-1}}\right)\right]\left[\tilde{Q}(z)+O\left(\frac{t}{|n|^{\beta-1}}\right)\right] \\
= & \frac{1-z}{1-\tilde{\lambda}(z)} \tilde{P}(z)+(1-z)(I-\tilde{R}(z) \tilde{Q}(z))^{-1} \tilde{Q}(z)+O\left(\frac{t}{|n|^{\beta-1}}+1_{n \geqslant 0} t^{3}\left(1-\frac{d}{256} t^{2}\right)^{n}\right) \\
= & \left(\frac{I-\tilde{R}(z)}{1-z}\right)^{-1}+O\left(\frac{t}{|n|^{\beta-1}}+1_{n \geqslant 0} t^{3}\left(1-\frac{d}{256} t^{2}\right)^{n}\right) .
\end{aligned}
$$

Corollary 3.6. We have

$$
\left(\frac{I-R(z, t)}{1-(1-(1 / \mu) M(t)) z}\right)^{-1}=\left(\frac{I-R(z)}{1-z}\right)^{-1}+O\left(\frac{t}{|n|^{\beta-1}}+1_{n \geqslant 0} t^{3}\left(1-\frac{d}{512} t^{2}\right)^{n}\right) .
$$

Proof. Let $\chi_{1}, \chi_{2}$ be a $C^{\infty}$ partition of unity of $S^{1}$ such that $R(z)=\tilde{R}(z)$ on the support of $\chi_{1}$. Since $\chi_{1}$ is $C^{\infty}$, $\chi_{1}(z)=O\left(1 /|n|^{\beta-1}\right)$. Writing $A(z, t)=((I-R(z, t)) /(1-(1-(1 / \mu) M(t)) z))^{-1}$, Corollary 3.5 ensures that

$$
\chi_{1}(z) A(z, t)=\chi_{1}(z) A(z, 0)+O\left(\frac{t}{|n|^{\beta-1}}+1_{n \geqslant 0} t^{3}\left(1-\frac{d}{512} t^{2}\right)^{n}\right) .
$$

Concerning $\chi_{2}$, we can modify $R$ outside of its support so that $I-R(z)$ is everywhere invertible on $S^{1}$. Let $\mathcal{A}=$ $\mathcal{O}_{\beta-1}(\operatorname{Hom}(E))$. Since $I-R(z, t)=I-R(z)+O_{\mathcal{A}}(t)$, Theorem A.3 yields $(I-R(z, t))^{-1}=(I-R(z))^{-1}+$ $O_{\mathcal{A}}(t)$. Hence,

$$
\chi_{2}(z) A(z, t)=\chi_{2}(z) A(z, 0)+O\left(\frac{t}{|n|^{\beta-1}}\right) .
$$

In fact, the functions in the previous corollary are defined on the whole disk $\overline{\mathbb{D}}$, i.e. their coefficients for $n<0$ vanish. However, during the proof, it was important to work in a less restrictive context, for example to introduce partitions of unity. 
Proof of Theorem 3.1. Set

$$
F_{t}(z)=\left(\frac{I-R(z, t)}{1-(1-(1 / \mu) M(t)) z}\right)^{-1}-\left(\frac{I-R(z)}{1-z}\right)^{-1}
$$

and

$$
\left(\frac{I-R(z)}{1-z}\right)^{-1}=\frac{1}{\mu} P+(1-z) A(z)
$$

where $A(z)=O\left(1 / n^{\beta-1}\right)$, by [32, Theorem 1] or [16, Theorem 1.1].

Then

$$
\begin{aligned}
\sum T_{n}(t) z^{n} & =(I-R(z, t))^{-1} \\
& =\frac{1}{1-(1-(1 / \mu) M(t)) z} \frac{1}{\mu} P+\frac{1-z}{1-(1-(1 / \mu) M(t)) z} A(z)+\frac{1}{1-(1-(1 / \mu) M(t)) z} F_{t}(z) \\
& =\mathrm{I}+\mathrm{II}+\mathrm{III} .
\end{aligned}
$$

The coefficient of $z^{n}$ in I is $\frac{1}{\mu}\left(1-\frac{1}{\mu} M(t)\right)^{n} P$. So, we have to bound the coefficients of II and III to conclude.

By (9) and Lemma 3.2, the coefficients of II belong to

$$
\left[1+O\left(t^{2}\left(1-d t^{2}\right)^{n}\right)\right] \star O\left(\frac{1}{n^{\beta-1}}\right) \subset O\left(\frac{1}{n^{\beta-1}}+t^{2}\left(1-\frac{d}{2} t^{2}\right)^{n}\right) .
$$

For III, we get by Corollary 3.6 that the coefficient of $z^{n}$ is bounded by

$$
O\left(\left(1-d t^{2}\right)^{n}\right) \star O\left(\frac{t}{n^{\beta-1}}+t^{3}\left(1-\frac{d}{512} t^{2}\right)^{n}\right) .
$$

The convolution between $\left(1-d t^{2}\right)^{n}$ and $t^{3}\left(1-\frac{d}{512} t^{2}\right)^{n}$ is bounded by the convolution between $\left(1-\frac{d}{512} t^{2}\right)^{n}$ and $t^{3}\left(1-\frac{d}{512} t^{2}\right)^{n}$, which gives $n t^{3}\left(1-\frac{d}{512} t^{2}\right)^{n}$. This is less than $C t\left(1-\frac{d}{1024} t^{2}\right)^{n}$, since

$$
\frac{n}{2}\left(1-c t^{2}\right)^{n} \leqslant \sum_{k=0}^{n / 2}\left(1-c t^{2}\right)^{k}\left(1-c t^{2}\right)^{n / 2} \leqslant\left(1-c t^{2}\right)^{n / 2} \frac{1}{c t^{2}} .
$$

As $t\left(1-\frac{d}{1024} t^{2}\right)^{n} \leqslant t\left(1-\frac{d}{1024} t^{2}\right)^{n} \star\left(\frac{1}{n^{\beta-1}}\right)$, we get a bound of the form stated in the theorem.

\section{The key estimate}

In this section, the assumptions are as in Theorem 1.1, i.e. $X$ is a Young tower whose return time $\varphi$ satisfies $m[\varphi>n]=O\left(1 / n^{\beta}\right)$ with $\beta>2$. We also assume that $\operatorname{gcd}\left(\varphi_{i}\right)=1$. Take also $f \in C_{\tau}(X)$ with $\int f \mathrm{~d} m=0$.

The goal of this section is to estimate precisely $\int_{X} \mathrm{e}^{\mathrm{i} t S_{n} f} \cdot u \cdot v \circ T^{n} \mathrm{~d} m$ when $u, v$ are functions on $X$. We will use the same kind of perturbative ideas as in [30] and [17], but applied to transfer operators associated to induced maps on the basis $B$ of the tower (see [32]). Separating the different return times, it will be possible to use the abstract Theorem 3.1, to finally get the key estimate Theorem 4.6.

\subsection{First return transfer operators}

Let $\hat{T}$ be the transfer operator associated to $T$, defined by $\int u \cdot v \circ T \mathrm{~d} m=\int \hat{T} u \cdot v \mathrm{~d} m$. When $u$ is integrable on $X$, it is given by

$$
\hat{T}^{n} u(x)=\sum_{T^{n} y=x} g_{m}^{(n)}(y) u(y),
$$

where $g_{m}^{(n)}$ is the inverse of the jacobian of $T^{n}$ at $y$. 
As the basis $B$ of the tower plays a particular role, we will decompose the trajectories of the preimages of $x$ under $T^{n}$, keeping track of the moments their iterates fall again in $B$. More formally, we introduce the following operators:

$$
\begin{aligned}
& R_{n} u(x)=\sum_{\substack{T^{n} y=x \\
y \in B, T y, \ldots, T^{n-1} y \notin B, T^{n} y \in B}} g_{m}^{(n)}(y) u(y), \\
& T_{n} u(x)=\sum_{\substack{T^{n} y=x \\
y \in B, T^{n} y \in B}} g_{m}^{(n)}(y) u(y), \\
& A_{n} u(x)=\sum_{\substack{T^{n} y=x \\
y \in B, T y, \ldots, T^{n} y \notin B}} g_{m}^{(n)}(y) u(y), \\
& B_{n} u(x)=\sum_{\substack{T^{n} y=x \\
y, \ldots, T^{n-1} y \notin B, T^{n} y \in B}} g_{m}^{(n)}(y) u(y), \\
& C_{n} u(x)=\sum_{\substack{T^{n} y=x \\
y, \ldots, T^{n} y \notin B}} g_{m}^{(n)}(y) u(y) .
\end{aligned}
$$

The interpretations of these operators are as follows: $R_{n}$ takes into account the first returns to $B$, while $T_{n}$ takes all returns into account. Hence,

$$
T_{n}=\sum_{k_{1}+\cdots+k_{l}=n} R_{k_{1}} \cdots R_{k_{l}}
$$

The operators $B_{n}$ and $A_{n}$ see respectively the beginning and the end of the trajectories, outside of $B$. Thus, if $x$ is fixed and $y$ satisfies $T^{n} y=x$ and $\left\{y, T y, \ldots, T^{n} y\right\} \cap B \neq \emptyset$, we can consider the first $b$ iterates of $y$, until it enters in $B$ (this corresponds to $B_{b}$ ), then some successive returns to $B$, during a time $k$ (this corresponds to $T_{k}$ ), and finally $a$ iterations outside of $B$ (corresponding to $A_{a}$ ). Thus,

$$
\hat{T}^{n}=C_{n}+\sum_{a+k+b=n} A_{a} T_{k} B_{b} .
$$

The operator $C_{n}$ takes into account the points $y$ such that $\left\{y, T y, \ldots, T^{n} y\right\} \cap B=\emptyset$.

We perturb these operators, setting (for $X=A, B, C, R, T$, and $t \in \mathbb{R}$ )

$$
X_{n}(t)(\cdot)=X_{n}\left(\mathrm{e}^{\mathrm{i} t S_{n} f} \cdot\right) .
$$

Eq. (10) remains true with $t$ everywhere:

$$
T_{n}(t)=\sum_{k_{1}+\cdots+k_{l}=n} R_{k_{1}}(t) \cdots R_{k_{l}}(t) .
$$

Let $\hat{T}(t)$ be the perturbation of $\hat{T}$ given by $\hat{T}(t)(\cdot)=\hat{T}\left(\mathrm{e}^{\mathrm{i} t f} \cdot\right)$. Then the following analogue of (11) holds:

$$
\hat{T}(t)^{n}=C_{n}(t)+\sum_{a+k+b=n} A_{a}(t) T_{k}(t) B_{b}(t) .
$$

Let $f_{B}$ be given by (1). As $f_{B}=S_{n} f$ on $\{y \in B \mid \varphi(y)=n\}$, we get $R_{n}(t)(u)=R_{n}\left(\mathrm{e}^{\mathrm{i} t f_{B}} u\right)$. 
For $z \in \overline{\mathbb{D}}$, write $R(z)=\sum R_{n} z^{n}$, and

$$
R(z, t)=\sum_{n=0}^{\infty} R_{n}(t) z^{n} .
$$

Let $T_{B}$ be the first return map induced by $T$ on $B$, i.e. $T_{B}(x)=T^{\varphi(x)}(x)$. Then (14) corresponds to considering all the preimages of a point in $B$ under $T_{B}$, whence $R(1)$ is the transfer operator $\hat{T}_{B}$ associated to $T_{B}$, and

$$
R(1, t)(u)=\hat{T}_{B}\left(\mathrm{e}^{\mathrm{i} t f_{B}} u\right) .
$$

When $u$ is a function on a subset $Z$ of $X$, we denote by $D_{\tau} u(Z)$ the best Hölder constant of $u$ on $Z$, i.e.

$$
D_{\tau} u(Z)=\inf \left\{C>0|\forall x, y \in Z,| u(x)-u(y) \mid \leqslant C \tau^{s(x, y)}\right\}
$$

where $s(x, y)$ is the separation time of $x$ and $y$.

The operators $R_{n}$ and $R_{n}(t)$ act on $C_{\tau^{\prime}}(B)$ for any $\tau \leqslant \tau^{\prime}<1$. Take $\eta$ such that

$$
0<\eta<\min (1 / 2, \beta-2)
$$

and set $v=\tau^{\eta}$. For technical reasons, we will let the operators act on $C_{v}(B)$. We regroup in the following lemma all the estimates we will need later. Their proofs are rather straightforward, but sometimes lengthy. Hence, we will not give the details of the proofs, and rather give references to articles where similar estimates are proved.

Lemma 4.1. The operators $R_{n}$ and $R_{n}(t)$ acting on $C_{v}(B)$ satisfy the following estimates:

(1) $\sum_{k=n+1}^{\infty}\left\|R_{k}\right\|=O\left(1 / n^{\beta}\right)$.

(2) The operator $R(z, t)$ satisfies a Doeblin-Fortet inequality

$$
\left\|R(z, t)^{n} u\right\| \leqslant C(1+|t|)(v|z|)^{n}\|u\|+C|z|^{n}\|u\|_{L^{1}} .
$$

In particular, the spectral radius of $R(z, t)$ is $\leqslant|z|$, and its essential spectral radius is $\leqslant v|z|$. Thus, $I-R(z, t)$ is not invertible if and only if 1 is an eigenvalue of $R(z, t)$, and this can happen only for $|z|=1$.

(3) $R(1)$ has a simple isolated eigenvalue at 1 (the eigenspace is the space of constant functions), and $I-R(z)$ is invertible for $z \neq 1$.

(4) There exists $C>0$ such that, for any $t \in \mathbb{R}$, for every $n \in \mathbb{N}^{*}$,

$$
\sum_{k=n+1}^{\infty}\left\|R_{k}(t)-R_{k}(0)\right\| \leqslant C \frac{|t|}{n^{\beta-1}} .
$$

(5) For every $t \in \mathbb{R}$, there exists $C=C(t)$ such that, for any $t^{\prime} \in \mathbb{R}$, for every $n \in \mathbb{N}^{*},\left\|R_{n}\left(t^{\prime}\right)-R_{n}(t)\right\| \leqslant$ $C\left|t^{\prime}-t\right|^{1 / 2} / n^{\beta-1}$.

Proof. The first assertion is a consequence of [14, Lemma 4.2]: it gives $\left\|R_{n}\right\|=O(m[\varphi=n])$.

The inequality (18) is similar to [3, Proposition 2.1]. In this article, the hypothesis is that $D_{\nu} f_{B}(B)<\infty$, but the proofs work in fact as soon as $\sum m\left(B_{i, 0}\right) D_{v} f_{B}\left(B_{i, 0}\right)<\infty$. In our case, $D_{v} f_{B}\left(B_{i, 0}\right) \leqslant C \varphi_{i}$, whence $\sum m\left(B_{i, 0}\right) D_{v} f_{B}\left(B_{i, 0}\right) \leqslant C \sum m\left(B_{i, 0}\right) \varphi\left(B_{i, 0}\right)=C$ by Kac's Formula. Since the injection $C_{v}(B) \rightarrow L^{1}(B)$ is compact, the statement on the essential spectral radius of $R(z, t)$ is then a consequence of Hennion's Theorem [18].

The third assertion is [14, Lemma 4.3].

The two remaining assertions are proved by direct estimates, similar to the estimates in [3, Theorem 2.4]. The last one holds in $C_{\sqrt{\tau}}(X)$ but not in $C_{\tau}(X)$. This is the reason of the requirement $v \geqslant \sqrt{\tau}$.

We will also need the following estimates on $A_{a}(t), B_{b}(t)$ and $C_{n}(t)$, acting on $C_{v}(X)$. 
Lemma 4.2. Let $u, v \in L^{\infty}(X)$. There exists a constant $C$ such that, for any $t \in \mathbb{R}$, for any $a \in \mathbb{N}$,

$$
\left|\int A_{a}(t)(u) v-\int A_{a}(u) v\right| \leqslant C \frac{|t|}{a^{\beta-1}}\|u\|_{\infty}\|v\|_{\infty} .
$$

Moreover, $\int A_{a}(u) v=O\left(1 / a^{\beta}\right)$, and

$$
\sum_{a=0}^{\infty} \int A_{a}\left(1_{B}\right) v=\int v .
$$

Proof. Define a function $v^{\prime}$ on $B$ by $v^{\prime}(x)=v\left(T^{a} x\right)$ if $\varphi(x)>a$, and 0 otherwise. Changing variables, we get $\int A_{a}(t)(u) v=\int_{\{\varphi>a\}} \mathrm{e}^{\mathrm{i} t S_{a} f} u v^{\prime}$. Since $\left|\mathrm{e}^{\mathrm{i} t S_{a} f}-1\right| \leqslant|t| a\|f\|_{\infty}$ and $m(\varphi>a) \leqslant C / a^{\beta}$, this implies (19).

Moreover, $\left|\int A_{a}(u) v\right| \leqslant m(\varphi>a)\|u\|_{\infty}\|v\|_{\infty} \leqslant\left(C / a^{\beta}\right)\|u\|_{\infty}\|v\|_{\infty}$. Finally, $\int A_{a}\left(1_{B}\right) v=\int_{T^{a}\{\varphi>a\}} v$. Since the sets $T^{a}\{\varphi>a\}$ form a partition of $X$, (20) readily follows.

Lemma 4.3. For $t \in \mathbb{R}, B_{b}(t)=B_{b}+O\left(|t| / b^{\beta-1}\right)$, where $\left\|B_{b}\right\|=O\left(1 / b^{\beta}\right)$ and $\forall u \in C_{v}(X)$,

$$
\sum_{b=0}^{\infty} \int_{B} B_{b} u \mathrm{~d} m=\int_{X} u \mathrm{~d} m .
$$

Proof. Let $\Lambda_{b}$ be the set of points that enter into $B$ after exactly $b$ iterations, so that $B_{b}(t)(u)$ takes the values of $u$ on $\Lambda_{b}$ into account. As in [3, Theorem 2.4], we check that $\left\|B_{b}(t)-B_{b}\right\| \leqslant C|t| m\left(\Lambda_{b}\right) D_{v} S_{b} f\left(\Lambda_{b}\right)$. As $D_{v} S_{b} f \leqslant C b$ and $m\left(\Lambda_{b}\right)=m[\varphi>b]=O\left(1 / b^{\beta}\right)$, we get indeed that $\left\|B_{b}(t)-B_{b}\right\| \leqslant C|t| / b^{\beta-1}$.

Moreover, we check as in [14, Lemma 4.2.] that $\left\|B_{b}\right\|=O\left(m\left(\Lambda_{b}\right)\right)=O\left(1 / b^{\beta}\right)$. Finally, as $\int_{B} B_{b} u \mathrm{~d} m=$ $\int_{\Lambda_{b}} u \mathrm{~d} m$ we get $\sum_{b} \int_{B} B_{b} u \mathrm{~d} m=\int_{X} u \mathrm{~d} m$.

Lemma 4.4. Let $u \in L^{\infty}(X)$. There exists a constant $C>0$ such that, for any $t \in \mathbb{R}$, for any $n \in \mathbb{N}$,

$$
\left\|C_{n}(t)(u)\right\|_{L^{1}} \leqslant \frac{C}{n^{\beta-1}}\|u\|_{\infty} .
$$

Proof. Set $X_{n+1}=X \backslash \bigcup_{i=0}^{n} T^{-i} B$ and $Z_{n+1}=T^{n}\left(X_{n+1}\right)$. The function $C_{n}(u)$ vanishes outside of $Z_{n+1}$. Let $x \in Z_{n+1}$ and let $y$ be its preimage under $T^{n}$. Then $\left|C_{n}(t)(u)(x)\right|=|u(y)| \leqslant\|u\|_{\infty}$. Hence, $\left\|C_{n}(t)(u)\right\|_{L^{1}} \leqslant$ $m\left(Z_{n+1}\right)\|u\|_{\infty}$. Finally, $m\left(Z_{n+1}\right)=m\left(X_{n+1}\right)=\sum_{p=n+1}^{\infty} m(\varphi>p)=O\left(1 / n^{\beta-1}\right)$.

\subsection{Result for the induced map}

Eq. (15) and (2) in Lemma 4.1 imply that the perturbed transfer operator $R(1, t)=\hat{T}_{B}(t)=\hat{T}_{B}\left(\mathrm{e}^{\mathrm{i} t f_{B}} \cdot\right)$ has a spectral gap. Thus, the classical methods of [17] apply to it, and yield an asymptotic expansion of the maximal eigenvalue of $\hat{T}_{B}(t)$ (this implies a central limit theorem for $T_{B}$ and the function $f_{B}$, but we are not interested in it here). To estimate the speed in the central limit theorem, we will need a rather precise asymptotic expansion of this eigenvalue, given by the following proposition.

Proposition 4.5. Assume that $T$ and $f$ satisfy the assumptions of Theorem 1.1 in a mixing Young tower. Let $\sigma^{2}$ be the variance in this theorem. Then, for small enough $t, R(1, t)$ has a unique eigenvalue $\lambda(1, t)$ close to 1 . It can be written as $\lambda(1, t)=1-\frac{\sigma^{2}}{2 m(B)} L(t)$ for a function $L$ such that $L(t) \sim t^{2}$ when $t \rightarrow 0$.

Write $E_{B}(u)=\frac{1}{m(B)} \int_{B} u$, and define a function $a$ on $B$ by $a=\left(I-\hat{T}_{B}\right)^{-1}\left(\hat{T}_{B} f_{B}\right)$. Then

$$
\lambda(1, t)=E_{B}\left(\mathrm{e}^{\mathrm{i} t f_{B}}\right)-t^{2} E_{B}\left(a f_{B}\right)+O\left(t^{3}\right) .
$$


Proof. The fact that $\lambda(1, t)=1-\frac{\sigma^{2}}{2 m(B)}\left(t^{2}+o\left(t^{2}\right)\right)$ is a consequence of [14, Theorem 3.7]. It remains to prove (21). As everything takes place in $B$, we can multiply $m$ by a constant, and assume that $m(B)=1$. Set $R_{t}=R(1, t)$, and let $\xi_{t}$ be the eigenfunction of $R_{t}$ corresponding to its eigenvalue $\lambda_{t}$ close to 1 . We normalize it so that $\int \xi_{t}=1$. We will also write $R=R_{0}=\hat{T}_{B}$.

Lemma 3.4 of [14] states that there exists a constant $C$ such that, if $g: B \rightarrow \mathbb{R}$ is integrable,

$$
\|R g\|_{\nu} \leqslant C\left(\|g\|_{L^{1}}+\sum_{I} m\left(B_{i, 0}\right) D_{\nu} g\left(B_{i, 0}\right)\right)
$$

where $D_{\nu} g\left(B_{i, 0}\right)$ is the best $\nu$-Hölder constant of $g$ on $B_{i, 0}$, defined in (16). In particular, $R f_{B} \in C_{v}(B)$.

Let us show that, in $C_{v}(B)$,

$$
R\left(\frac{\mathrm{e}^{\mathrm{i} t f_{B}}-1}{t}\right)=\mathrm{i} R\left(f_{B}\right)+O(t) .
$$

The Taylor Formula gives

$$
\frac{\mathrm{e}^{\mathrm{i} t f_{B}}-1}{t}-\mathrm{i} f_{B}=-t f_{B}^{2} \int_{0}^{1}(1-u) \mathrm{e}^{\mathrm{i} t u f_{B}} \mathrm{~d} u .
$$

We use this formula to bound $D_{v}\left(\frac{\mathrm{e} i f_{B}-1}{t}-\mathrm{i} f_{B}\right)\left(B_{i, 0}\right)$, where $B_{i, 0}$ is an element of the partition of $B$. Set $n=\varphi\left(B_{i, 0}\right)$ the return time on $B_{i, 0}$. As $D_{v}\left(h_{1} h_{2}\right) \leqslant D_{v}\left(h_{1}\right)\left\|h_{2}\right\|_{\infty}+\left\|h_{1}\right\|_{\infty} D_{v}\left(h_{2}\right)$,

$$
\begin{aligned}
& D_{\nu}\left(\frac{\mathrm{e}^{\mathrm{i} t f_{B}}-1}{t}-\mathrm{i} f_{B}\right)\left(B_{i, 0}\right) \\
& \quad \leqslant|t|\left\|\left(f_{B}\right)_{\mid B_{i, 0}}\right\|_{\infty} D_{\nu} f_{B}\left(B_{i, 0}\right)+|t|\left\|\left(f_{B}\right)_{\mid B_{i, 0}}\right\|_{\infty}^{2} \int_{0}^{1} D_{\nu}\left(\mathrm{e}^{\mathrm{i} t u f_{B}}\right)\left(B_{i, 0}\right) \mathrm{d} u .
\end{aligned}
$$

The first term is $\leqslant|t| n^{2}$. For the second term, take $C$ such that $\left|\mathrm{e}^{\mathrm{i} s}-1\right| \leqslant C|s|^{\eta}$ for any $s \in \mathbb{R}$ (where $\eta$ was defined in (17)). Then, if $x, y \in B_{i, 0}$,

$$
\begin{aligned}
\left|\mathrm{e}^{\mathrm{i} t u f_{B}(x)}-\mathrm{e}^{\mathrm{i} t u f_{B}(y)}\right| & =\left|\mathrm{e}^{\mathrm{i} t u\left(f_{B}(x)-f_{B}(y)\right)}-1\right| \leqslant C\left|t u\left(f_{B}(x)-f_{B}(y)\right)\right|^{\eta} \\
& \leqslant C\left|D_{\tau} f_{B}\left(B_{i, 0}\right) \tau^{s(x, y)}\right|^{\eta} \leqslant C n^{\eta} \nu^{s(x, y)},
\end{aligned}
$$

whence $D_{v}\left(\mathrm{e}^{\mathrm{i} t u f_{B}}\right)\left(B_{i, 0}\right) \leqslant C n^{\eta}$. An integration yields

$$
D_{v}\left(\frac{\mathrm{e}^{\mathrm{i} t f_{B}}-1}{t}-\mathrm{i} f_{B}\right)\left(B_{i, 0}\right) \leqslant|t| n^{2+\eta} .
$$

Eq. (22) gives that

$$
\left\|R\left(\frac{\mathrm{e}^{\mathrm{i} t f_{B}}-1}{t}-\mathrm{i} f_{B}\right)\right\|_{\nu} \leqslant C\left(\left\|\frac{\mathrm{e}^{\mathrm{i} t f_{B}}-1}{t}-\mathrm{i} f_{B}\right\|_{L^{1}}+\sum m[\varphi=n]|t| n^{2+\eta}\right) .
$$

As $\left|\mathrm{e}^{\mathrm{i} t f_{B}}-1-\mathrm{i} t f_{B}\right| \leqslant t^{2} f_{B}^{2}$, with $f_{B}^{2}$ integrable, the first term is $O(t)$. For the second term, $\sum m[\varphi=n] n^{2+\eta}=$ $\sum(m[\varphi>n-1]-m[\varphi>n]) n^{2+\eta} \leqslant C \sum m[\varphi>n] n^{1+\eta}$. Since $m[\varphi>n]=O\left(1 / n^{\beta}\right)$, this sum is finite by definition of $\eta$. This proves (23).

We return to the study of the eigenvalue $\lambda_{t}$. As $\lambda_{t} \xi_{t}=R_{t} \xi_{t}$, we get after integration that

$$
\lambda_{t}=E\left(\mathrm{e}^{\mathrm{i} t f_{B}}\right)+\int\left(\mathrm{e}^{\mathrm{i} t f_{B}}-1\right)\left(\xi_{t}-1\right)
$$


As $f_{B} \in L^{2}$ and $\int f_{B}=0$, we have

$$
E\left(\mathrm{e}^{\mathrm{i} t f_{B}}\right)=1+\mathrm{i} t \int f_{B}-\frac{t^{2}}{2} \int f_{B}^{2}+o\left(t^{2}\right)=1-\frac{t^{2}}{2} \int f_{B}^{2}+o\left(t^{2}\right) .
$$

Moreover, by (4) in Lemma 4.1, $\xi_{t}-1=O\left(\left\|R_{t}-R\right\|\right)=O(t)$ in $C_{v}(B)$, hence in $L^{2}$, and $\mathrm{e}^{\mathrm{i} t f_{B}}-1=\mathrm{i} t f_{B}+o(t)=$ $O(t)$ in $L^{2}$. Consequently, $\int\left(\mathrm{e}^{\mathrm{i} t f_{B}}-1\right)\left(\xi_{t}-1\right)=O\left(t^{2}\right)$, which implies that $\lambda_{t}=1+O\left(t^{2}\right)$.

Thus,

$$
\frac{\xi_{t}-1}{t}=\frac{\lambda_{t} \xi_{t}-\xi_{0}}{t}+O(t)=\left(R_{t}-R_{0}\right) \frac{\xi_{t}-\xi_{0}}{t}+R \frac{\xi_{t}-\xi_{0}}{t}+\frac{R_{t}-R_{0}}{t} \xi_{0}+O(t) .
$$

As $R_{t}-R_{0}=O(t)$ and $\left(\xi_{t}-\xi_{0}\right) / t$ is bounded, $\left(R_{t}-R_{0}\right)\left(\xi_{t}-\xi_{0}\right) / t=O(t)$. Moreover, $\left(\left(R_{t}-R_{0}\right) / t\right) \xi_{0}=$ $R\left(\left(\mathrm{e}^{\mathrm{i} t f_{B}}-1\right) / t\right)$. Hence,

$$
(I-R) \frac{\xi_{t}-\xi_{0}}{t}=R\left(\frac{\mathrm{e}^{\mathrm{i} t f_{B}}-1}{t}\right)+O(t) .
$$

As $R f_{B} \in C_{v}(B)$, and $I-R$ is invertible on the functions of $C_{v}(B)$ with vanishing integral ((3) in Lemma 4.1), it is possible to define $a=(I-R)^{-1}\left(R f_{B}\right) \in C_{v}(B)$. Then, using (23),

$$
(I-R)\left(\frac{\xi_{t}-\xi_{0}}{t}-\mathrm{i} a\right)=R\left(\frac{\mathrm{e}^{\mathrm{i} t f_{B}}-1}{t}-\mathrm{i} f_{B}\right)+O(t)=O(t) .
$$

As the inverse of $I-R$ is continuous on the functions with zero integral, we get that, in $C_{v}(B)$ (hence in $L^{\infty}$ ),

$$
\xi_{t}=1+t \mathrm{i} a+O\left(t^{2}\right) .
$$

As $\mathrm{e}^{\mathrm{i} t f_{B}}=1+\mathrm{i} t f_{B}+O\left(t^{2}\right)$ in $L^{1}$ since $f_{B}^{2}$ is integrable, we get

$$
\int\left(\mathrm{e}^{\mathrm{i} t f_{B}}-1\right)\left(\xi_{t}-1\right)=-t^{2} \int f_{B} a+O\left(t^{3}\right) .
$$

Eq. (24) yields the desired conclusion.

\subsection{The key estimate}

Theorem 4.6. Let $X$ be a Young tower with $m[\varphi>n]=O\left(1 / n^{\beta}\right)$ for $\beta>2$, and $\operatorname{gcd}\left(\varphi_{i}\right)=1$. Let $f \in C_{\tau}(X)$ be of zero integral. Let $\sigma$ and $L(t)$ be given by Proposition 4.5, and assume $\sigma>0$. Then there exist $\alpha>0, C>0$ and $d>0$ such that, for any $u \in C_{\tau}(X)$ and $v \in L^{\infty}(X)$, for any $n \in \mathbb{N}^{*}$, for any $t \in[-\alpha, \alpha]$,

$$
\begin{aligned}
& \left|\int_{X} \mathrm{e}^{\mathrm{i} t S_{n} f} \cdot u \cdot v \circ T^{n} \mathrm{~d} m-\left(1-\frac{\sigma^{2}}{2} L(t)\right)^{n}\left(\int_{X} u \mathrm{~d} m\right)\left(\int_{X} v \mathrm{~d} m\right)\right| \\
& \quad \leqslant C\left[\frac{1}{n^{\beta-1}}+|t|\left(\frac{1}{n^{\beta-1}}\right) \star\left(1-d t^{2}\right)^{n}\right]\|u\|\|v\|_{\infty} .
\end{aligned}
$$

Proof. Set $R(z, t)=\sum R_{n}(t) z^{n}$, we want to apply Theorem 3.1 to $R(z, t)$. Proposition 4.5 gives the behavior of the eigenvalue of $R(1, t)$, while Lemma 4.1 shows the required estimates. Finally, the spectral projection $P$ of $R(1,0)$ is the projection on the constant functions on $B$, given by $P g=\left(\int_{B} g \mathrm{~d} m\right) / m(B)$. It satisfies $P R^{\prime}(1) P=\frac{1}{m(B)} P$ [14, Lemma 4.4].

Consequently, we can apply Theorem 3.1 with $M(t)=\frac{\sigma^{2}}{2 m(B)} L(t)$ and $\mu=\frac{1}{m(B)}$. As $\sum T_{n}(t) z^{n}=(I-$ $R(z, t))^{-1}$ by (12), we get that there exists an error term $E(n, t)$ such that $T_{n}(t)=m(B)\left(1-\frac{\sigma^{2}}{2} L(t)\right)^{n} P+E(n, t)$, with

$$
\|E(n, t)\| \leqslant C\left[\frac{1}{n^{\beta-1}}+|t|\left(\frac{1}{n^{\beta-1}}\right) \star\left(1-d t^{2}\right)^{n}\right]=: e(n, t) .
$$


In the following, $C$ and $d$ will denote generic constants, that may vary finitely many times. In particular, we may write inequalities like $10 e(n, t) \leqslant e(n, t)$. With this convention, Lemma 3.2 implies that

$$
\left(\frac{1}{n^{\beta-1}}\right) \star e(n, t)=O(e(n, t)) .
$$

Let $u \in C_{\tau}(X)$ and $v \in L^{\infty}(X)$. To simplify the expressions, we will assume that these functions are of norm at most 1. Then (13) implies that

$$
\begin{aligned}
\int_{X} \mathrm{e}^{\mathrm{i} t S_{n} f} \cdot u \cdot v \circ T^{n}= & \int_{X} \hat{T}^{n}\left(\mathrm{e}^{\mathrm{i} t S_{n} f} u\right) v=\int_{X} \hat{T}(t)^{n}(u) v \\
= & \sum_{a+k+b=n} \int_{X} A_{a}(t) T_{k}(t) B_{b}(t)(u) v+\int_{X} C_{n}(t)(u) v \\
= & \sum_{a+k+b=n} \int_{X} A_{a}(t) m(B)\left(1-\frac{\sigma^{2}}{2} L(t)\right)^{k} P B_{b}(t)(u) v \\
& +\sum_{a+k+b=n} \int_{X} A_{a}(t) E(k, t) B_{b}(t)(u) v+\int_{X} C_{n}(t)(u) v .
\end{aligned}
$$

By Lemma $4.4,\left|\int_{X} C_{n}(t)(u) v\right| \leqslant C / n^{\beta-1} \leqslant e(n, t)$.

Let us bound the second sum of (28). If $h \in C_{v}(X)$, the function $A_{a}(t) h$ is supported in $T^{a}\{y \in B \mid$ $\varphi(y)>a\}$, whose measure is $m[\varphi>a]=O\left(1 / a^{\beta}\right)$. Thus, $\left|\int_{X} A_{a}(t) h\right| \leqslant\left(C / a^{\beta}\right)\|h\|_{\infty} \leqslant\left(C / a^{\beta-1}\right)\|h\|$. Moreover, $\left\|E(k, t) B_{b}(t)(u)\right\| \leqslant e(k, t)\left\|B_{b}(t)\right\| \leqslant e(k, t) C / b^{\beta-1}$ by Lemma 4.3. Thus,

$$
\begin{aligned}
\left|\sum_{a+k+b=n} \int_{X} A_{a}(t) E(k, t) B_{b}(t)(u) v\right| & \leqslant \sum_{a+k+b=n} \frac{C}{a^{\beta-1}} e(k, t) \frac{C}{b^{\beta-1}} \\
& \leqslant C\left(\frac{1}{n^{\beta-1}}\right) \star e(n, t) \star\left(\frac{1}{n^{\beta-1}}\right) \leqslant e(n, t)
\end{aligned}
$$

by (27).

The first sum of (28) can be written as

$$
I=\sum_{a+k+b=n}\left(\int_{X} A_{a}(t)\left(1_{B}\right) v\right)\left(1-\frac{\sigma^{2}}{2} L(t)\right)^{k}\left(\int_{B} B_{b}(t)(u)\right) .
$$

Using Lemmas 4.2 and 4.3 and convolving, we find a sequence $w_{n}$ such that $w_{n}=O\left(1 / n^{\beta}\right), \sum w_{n}=\left(\int u\right)\left(\int v\right)$, and

$$
I=\left(w_{n}+O\left(\frac{|t|}{n^{\beta-1}}\right)\right) \star\left(1-\frac{\sigma^{2}}{2} L(t)\right)^{k} .
$$

As $L(t) \sim t^{2}$ when $t \rightarrow 0$, the term coming from $O\left(|t| / n^{\beta-1}\right)$ is bounded by $|t|\left(1 / n^{\beta-1}\right) \star\left(1-d t^{2}\right)^{n} \leqslant e(n, t)$. Moreover, for $x, y \in \mathbb{R}$,

$$
\left|\mathrm{e}^{x}-\mathrm{e}^{y}\right| \leqslant|x-y| \mathrm{e}^{\max (x, y)} .
$$

Thus, 


$$
\begin{aligned}
& \left|\sum_{k=0}^{n} w_{n-k}\left(1-\frac{\sigma^{2}}{2} L(t)\right)^{k}-\sum_{k=0}^{n} w_{n-k}\left(1-\frac{\sigma^{2}}{2} L(t)\right)^{n}\right| \\
& \quad \leqslant \sum_{k=0}^{n} \frac{C}{(n-k)^{\beta}}(n-k)\left|\ln \left(1-\frac{\sigma^{2}}{2} L(t)\right)\right|\left(1-\frac{\sigma^{2}}{2} L(t)\right)^{k} \\
& \quad \leqslant \sum_{k=0}^{n} C t^{2}\left(1-d t^{2}\right)^{k} \frac{1}{(n-k)^{\beta-1}} \leqslant e(n, t) .
\end{aligned}
$$

Hence, up to $O(e(n, t))$, the integral $\int \mathrm{e}^{\mathrm{i} t S_{n} f} \cdot u \cdot v \circ T^{n}$ is equal to $\sum_{k=0}^{n} w_{n-k}\left(1-\frac{\sigma^{2}}{2} L(t)\right)^{n}$. Finally,

$$
\begin{aligned}
& \left|\left(1-\frac{\sigma^{2}}{2} L(t)\right)^{n} \int_{X} u \int_{X} v-\sum_{k=0}^{n} w_{n-k}\left(1-\frac{\sigma^{2}}{2} L(t)\right)^{n}\right| \\
& \quad=\left(1-\frac{\sigma^{2}}{2} L(t)\right)^{n}\left|\sum_{k=n+1}^{\infty} w_{k}\right| \leqslant C \sum_{k=n+1}^{\infty} \frac{1}{k^{\beta}} \leqslant \frac{C}{n^{\beta-1}} \leqslant e(n, t) .
\end{aligned}
$$

This concludes the proof.

When $u=v=1$, Theorem 4.6 states that, for small $t$, the characteristic function of $S_{n} f$ behaves essentially like $\left(1-\frac{\sigma^{2}}{2} L(t)\right)^{n}$, which is very similar to the characteristic function of the sum of $n$ independent identically distributed random variables. Hence, using this estimate, it will be possible to use the classical probabilistic proofs to get the local limit theorem or the Berry-Esseen theorem. However, some care is still required to check that the error term in Theorem 4.6 is sufficiently small so that these proofs still work.

\section{Proof of the local limit theorem}

\subsection{Periodicity problems}

This paragraph is related to the end of Section 3 of [3]. The differences come mainly from the inductive process and the fact that we are considering series of operators instead of a single operator.

Let $X$ be a Young tower with $\operatorname{gcd}\left(\varphi_{i}\right)=1, \tau<1$ and $f \in C_{\tau}(X)$. For $t \in \mathbb{R}$, $\mathrm{e}^{\mathrm{i} t f}$ is said to be cohomologous to a constant $\lambda \in S^{1}$ if there exists $\omega: X \rightarrow S^{1}$ such that $\mathrm{e}^{\mathrm{i} t f}=\lambda(\omega \circ T) / \omega$ almost everywhere.

Proposition 5.1. Let $t \in \mathbb{R}$, and $z \in S^{1}$. The following assertions are equivalent:

1. $\mathrm{e}^{\mathrm{i} t f}$ is cohomologous to $z^{-1}$.

2. 1 is an eigenvalue of the operator $R(z, t)$ acting on $C_{v}(B)$.

Proof. Suppose first that there exists a nonzero $\omega \in C_{v}(B)$ such that $R(z, t) \omega=\omega$. As $R(z, t)(v)=\hat{T}_{B}\left(z^{\varphi} \mathrm{e}^{\mathrm{i} t f_{B}} v\right)$, the adjoint operator of $R(z, t)$ in $L^{2}$ is $W(v)=z^{-\varphi} \mathrm{e}^{-\mathrm{i} t f_{B}} v \circ T_{B}$. Then $\|W \omega-\omega\|_{L^{2}}^{2}=\|W \omega\|_{L^{2}}^{2}-\|\omega\|_{L^{2}}^{2}$. As $T_{B}$ preserves the measure, we have $\|\omega\|_{L^{2}}=\|W \omega\|_{L^{2}}$, whence $\|W \omega-\omega\|_{L^{2}}=0$. Consequently, $\omega=z^{-\varphi} \mathrm{e}^{-\mathrm{i} t f_{B}} \omega \circ T_{B}$ almost everywhere. Taking the modulus, $|\omega|=|\omega| \circ T_{B}$, and the ergodicity of $T_{B}$ gives that $|\omega|$ is almost everywhere constant. We can assume that $|\omega|=1$. We extend the function $\omega$ to $X$ by setting

$$
\omega(x, k)=\omega(x, 0) \mathrm{e}^{\mathrm{i} t f(x, 0)} \cdots \mathrm{e}^{\mathrm{i} t f(x, k-1)} z^{k} .
$$


Then, for $k<\varphi(x)-1$, we have by construction $\omega \circ T(x, k) / \omega(x, k)=z \mathrm{e}^{\mathrm{i} t f(x, k)}$. Moreover, for $k=\varphi(x)-1$,

$$
\omega \circ T(x, k) / \omega(x, k)=\omega \circ T_{B}(x, 0) / \omega(x, k)=\mathrm{e}^{\mathrm{i} t f_{B}(x, 0)} z^{k+1} \omega(x, 0) / \omega(x, k)=\mathrm{e}^{\mathrm{i} t f(x, k)} z .
$$

Thus, $\mathrm{e}^{\mathrm{i} t f}=z^{-1} \omega \circ T / \omega$ almost everywhere.

Conversely, suppose that a measurable function $\omega$ satisfies $\mathrm{e}^{\mathrm{i} t f}=z^{-1} \omega \circ T / \omega$. The previous calculations give $\mathrm{e}^{\mathrm{i} t f_{B}}=z^{-\varphi} \omega \circ T_{B} / \omega$. The operator $R(z, t)=\hat{T}_{B}\left(z^{\varphi} \mathrm{e}^{\mathrm{i} t f_{B}} \cdot\right)$ acts on $L^{1}$, and satisfies

$$
R(z, t)(\omega)=\hat{T}_{B}\left(z^{\varphi} \mathrm{e}^{\mathrm{i} t f_{B}} \omega\right)=\hat{T}_{B}\left(\omega \circ T_{B}\right)=\omega .
$$

But, in Lemma 4.1, we have seen that $R(z, t)$ satisfies a Doeblin-Fortet inequality between the spaces $C_{v}(B)$ and $L^{1}(B)$. [20] ensures that the eigenfunctions of $R(z, t)$ in $L^{1}(B)$ for the eigenvalue 1 are in fact in $C_{v}(B)$, i.e. $\omega \in C_{v}(B)$.

Corollary 5.2. The set $\mathfrak{A}:=\left\{t \in \mathbb{R} \mid \mathrm{e}^{\mathrm{i} t f}\right.$ is cohomologous to a constant $\}$ is a closed subgroup of $\mathbb{R}$. Moreover, for every $t \in \mathfrak{A}$, there exists a unique $z(t) \in S^{1}$ such that $\mathrm{e}^{\mathrm{i} t f}$ is cohomologous to $z(t)$. Finally, the map $t \mapsto z(t)$ is a continuous morphism from $\mathfrak{A}$ to $S^{1}$.

Proof. The set $\mathfrak{A}$ is clearly a subgroup of $\mathbb{R}$. If $\mathrm{e}^{\mathrm{i} t f}$ is cohomologous simultaneously to $z$ and $z^{\prime}$, then $z^{\prime}=$ $\left(\mathrm{e}^{\mathrm{i} h \circ T} / \mathrm{e}^{\mathrm{i} h}\right) z$ for some function $h: X \rightarrow \mathbb{R}$. As $T$ is mixing [35, Theorem 1(iii)], the only constant $s$ satisfying $g \circ T=s g$ for some nonzero function $g$ is 1 . This implies that $z=z^{\prime}$. The map $t \mapsto z(t)$ is thus well defined, and it is clearly a group morphism.

It remains to check that $\mathfrak{A}$ is closed and that $z(t)$ is continuous. Let $t_{n}$ be a sequence of $\mathfrak{A}$ converging to $T \in \mathbb{R}$. Let $Z$ be a cluster point of the sequence $z\left(t_{n}\right)$. By (5) in Lemma 4.1, $(z, t) \mapsto R(z, t)$ is a continuous map with values in $\operatorname{Hom}\left(C_{v}(B)\right)$. If $I-R\left(Z^{-1}, T\right)$ were invertible, then $I-R\left(z_{n}^{-1}, t_{n}\right)$ would also be invertible for large enough $n$, which is a contradiction by the previous proposition. Thus, $I-R\left(Z^{-1}, T\right)$ is not invertible, whence 1 is an eigenvalue of $R\left(Z^{-1}, T\right)$ by quasi-compactness. This implies that $T \in \mathfrak{A}$ and $Z=z(T)$, once again by the previous proposition.

Consequently, there are three cases to be considered for the local limit theorem: $\mathfrak{A}$ is either $\mathbb{R}$, or $\{0\}$, or a discrete subgroup of $\mathbb{R}$. If it is $\mathbb{R}$, [25] ensures that $f$ can be written as $g-g \circ T$, hence $\sigma=0$ in the central limit theorem, and there is nothing to prove. If $\mathfrak{A}=\{0\}$, it is not possible to write $f$ as $\rho+g-g \circ T+\lambda q$, where $\rho \in \mathbb{R}$, $g: X \rightarrow \mathbb{R}$ is measurable, $\lambda>0$ and $q: X \rightarrow \mathbb{Z}$, i.e. $f$ is aperiodic. This case is handled by Theorem 1.2. Finally, $\mathfrak{A}=2 \pi \mathbb{Z}$ means that $f=\rho+g-g \circ T+q$ where $q$ takes integer values, and that there is no such expression where $q$ takes its values in $n \mathbb{Z}$ with $n \geqslant 2$. This is dealt with in Theorem 5.3.

\subsection{The aperiodic case}

Proof of Theorem 1.2. Let $f$ be an aperiodic function on $X$, as in the hypotheses of Theorem 1.2. Then, for every $(z, t) \in(\overline{\mathbb{D}} \times \mathbb{R})-\{(1,0)\}, I-R(z, t)$ is invertible: for $|z|<1$, the spectral radius of $R(z, t)$ is at most $|z|<1((2)$ in Lemma 4.1), and for $|z|=1$ this comes from Proposition 5.1 and Corollary 5.2, since $\mathfrak{A}=\{0\}$.

Let $\alpha>0$ be given by Theorem 4.6: we control the behavior of the integrals when $|t| \leqslant \alpha$. Take $K>0$. We will show the following fact: there exists $C>0$ such that, for every $|t| \in[\alpha, K]$, for every $n \in \mathbb{N}^{*}$, for all functions $u \in C_{\tau}(X)$ and $v \in L^{\infty}(X)$,

$$
\left|\int_{X} \mathrm{e}^{\mathrm{i} t S_{n} f} \cdot u \cdot v \circ T^{n}\right| \leqslant \frac{C}{n^{\beta-1}}\|u\|\|v\|_{\infty} .
$$

Let us write $\mathcal{A}=\mathcal{O}_{\beta-1}^{+}\left(\operatorname{Hom}\left(C_{v}(B)\right)\right)$ (the Banach algebra of series $\sum_{0}^{\infty} A_{n} z^{n}$ where $A_{n} \in \operatorname{Hom}\left(C_{v}(B)\right)$ and $\left\|A_{n}\right\|=O\left(1 / n^{\beta-1}\right)$, with the norm $\left.\left\|\sum A_{n} z^{n}\right\|=\sup _{n \in \mathbb{N}}(n+1)^{\beta-1}\left\|A_{n}\right\|\right)$. The map $t \mapsto R(z, t)$ is continuous from $[-K,-\alpha] \cup[\alpha, K]$ to $\mathcal{A}((5)$ in Lemma 4.1). Moreover, $I-R(z, t)$ is invertible on $\overline{\mathbb{D}}$ for $t$ in 
these intervals. Theorem A.4 shows that $(I-R(z, t))^{-1} \in \mathcal{A}$, and the continuity of the inversion even yields that $t \mapsto(I-R(z, t))^{-1}$ is continuous. By compactness, there exists $C$ such that $\left\|(I-R(z, t))^{-1}\right\|_{\mathcal{A}} \leqslant C$ for $|t| \in[\alpha, K]$. As $(I-R(z, t))^{-1}=\sum T_{n}(t) z^{n}$, this implies that $\left\|T_{n}(t)\right\| \leqslant C / n^{\beta-1}$, uniformly in $n$ and $t$.

We have

$$
\int_{X} \mathrm{e}^{\mathrm{i} t S_{n} f} \cdot u \cdot v \circ T^{n}=\int_{X} C_{n}(t)(u) v+\sum_{a+k+b=n} \int_{X} A_{a}(t) T_{k}(t) B_{b}(t)(u) v .
$$

By Lemma 4.4, $\left|\int_{X} C_{n}(t)(u) v\right| \leqslant\left(C / n^{\beta-1}\right)\|u\|_{\infty}\|v\|_{\infty}$. By Lemmas 4.2 and 4.3,

$$
\begin{aligned}
\left|\int A_{a}(t) T_{k}(t) B_{b}(t)(u) v\right| & \leqslant \frac{C}{a^{\beta-1}}\left\|T_{k}(t) B_{b}(t)(u)\right\|_{\infty}\|v\|_{\infty} \leqslant \frac{C}{a^{\beta-1}}\left\|T_{k}(t)\right\|\left\|B_{b}(t)\right\|\|u\|\|v\|_{\infty} \\
& \leqslant \frac{C}{a^{\beta-1}} \frac{C}{k^{\beta-1}} \frac{C}{b^{\beta-1}}\|u\|\|v\|_{\infty} .
\end{aligned}
$$

Thus,

$$
\left|\int_{X} \mathrm{e}^{\mathrm{i} t S_{n} f} \cdot u \cdot v \circ T^{n}\right| \leqslant C\left(\frac{1}{n^{\beta-1}}\right) \star\left(\frac{1}{n^{\beta-1}}\right) \star\left(\frac{1}{n^{\beta-1}}\right)\|u\|\|v\|_{\infty} \leqslant \frac{C}{n^{\beta-1}}\|u\|\|v\|_{\infty},
$$

and (31) is proved.

We prove now the local limit theorem, using the method of Breiman [7]. Take $u, v$ and $k_{n}$ as in the assumptions of Theorem 1.2. Let $\psi \in L^{1}(\mathbb{R})$ be such that its Fourier transform $\hat{\psi}$ is supported in $[-K, K]$. Then $\psi(x)=$ $\frac{1}{2 \pi} \int_{-K}^{K} \hat{\psi}(t) \mathrm{e}^{\mathrm{i} t x} \mathrm{~d} t$, whence

$$
\begin{aligned}
\sqrt{n} E\left(\psi\left(S_{n} f-k_{n}-u-v \circ T^{n}\right)\right)= & \frac{\sqrt{n}}{2 \pi} \int_{-K}^{K} \hat{\psi}(t) E\left(\mathrm{e}^{\mathrm{i} t\left(S_{n} f-k_{n}-u-v \circ T^{n}\right)}\right) \mathrm{d} t \\
= & \frac{\sqrt{n}}{2 \pi} \int_{-\alpha}^{\alpha} \hat{\psi}(t) \mathrm{e}^{-\mathrm{i} t k_{n}} E\left(\mathrm{e}^{\mathrm{i} t S_{n} f} \mathrm{e}^{-\mathrm{i} t u} \mathrm{e}^{-\mathrm{i} t v \circ T^{n}}\right) \mathrm{d} t \\
& +\frac{\sqrt{n}}{2 \pi} \int_{\alpha \leqslant|t| \leqslant K} \hat{\psi}(t) \mathrm{e}^{-\mathrm{i} t k_{n}} E\left(\mathrm{e}^{\mathrm{i} t S_{n} f} \mathrm{e}^{-\mathrm{i} t u} \mathrm{e}^{-\mathrm{i} t v \circ T^{n}}\right) \mathrm{d} t .
\end{aligned}
$$

For $\alpha \leqslant|t| \leqslant K$, the norms $\left\|\mathrm{e}^{-\mathrm{i} t u}\right\|$ and $\left\|\mathrm{e}^{-\mathrm{i} t v}\right\|_{\infty}$ remain bounded. Hence, (31) implies that $\mid E\left(\mathrm{e}^{\mathrm{i} t S_{n} f} \mathrm{e}^{-\mathrm{i} t u} \times\right.$ $\left.\mathrm{e}^{-\mathrm{i} t v \circ T^{n}}\right) \mid \leqslant C / n^{\beta-1}$. Therefore, the second integral tends to 0 . For the first integral, we approximate $E\left(\mathrm{e}^{\mathrm{i} t S_{n} f} \times\right.$ $\left.\mathrm{e}^{-\mathrm{i} t u} \mathrm{e}^{-\mathrm{i} t v \circ T^{n}}\right)$ by $\left(1-\frac{\sigma^{2}}{2} L(t)\right)^{n} \int \mathrm{e}^{-\mathrm{i} t u} \int \mathrm{e}^{-\mathrm{i} t v}$. By Theorem 4.6, the error term is bounded by

$$
C \sqrt{n} \int_{-\alpha}^{\alpha}\left(\frac{1}{n^{\beta-1}}+|t|\left(\frac{1}{n^{\beta-1}}\right) \star\left(1-d t^{2}\right)^{n}\right) \mathrm{d} t .
$$

Let us show that this integral tends to 0 . This is clear for the first term. For the second term, we cut the integral in two pieces. For $|t| \leqslant 1 / \sqrt{n}$, the convolution is bounded (since $\frac{1}{n^{\beta-1}}$ is summable and $\left(1-d t^{2}\right)^{n} \leqslant 1$ ), whence the integral is $\leqslant C \sqrt{n} \int_{|t| \leqslant 1 / \sqrt{n}}|t| \mathrm{d} t \rightarrow 0$. For $|t| \geqslant 1 / \sqrt{n}$, Lemma 3.2 gives that the convolution is bounded by $1 /\left(|t| n^{\beta-1}\right)+|t|\left(1-d t^{2}\right)^{n}$, whence the integral is less than

$$
C \sqrt{n} \int_{1 / \sqrt{n} \leqslant|t| \leqslant \alpha} \frac{1}{|t| n^{\beta-1}}+|t|\left(1-d t^{2}\right)^{n} \mathrm{~d} t \leqslant C \sqrt{n} \frac{\ln n}{n^{\beta-1}}+C \sqrt{n}\left[\frac{\left(1-d t^{2}\right)^{n+1}}{-2 d(n+1)}\right]_{1 / \sqrt{n}}^{\alpha} \rightarrow 0 .
$$


Finally, we have proved that

$$
\sqrt{n} E\left(\psi\left(S_{n} f-k_{n}-u-v \circ T^{n}\right)\right)=\frac{\sqrt{n}}{2 \pi} \int_{-\alpha}^{\alpha} \hat{\psi}(t) \mathrm{e}^{-\mathrm{i} t k_{n}}\left(1-\frac{\sigma^{2}}{2} L(t)\right)^{n} E\left(\mathrm{e}^{-\mathrm{i} t u}\right) E\left(\mathrm{e}^{-\mathrm{i} t v}\right) \mathrm{d} t+o(1) .
$$

But

$$
\begin{aligned}
& \frac{\sqrt{n}}{2 \pi} \int_{-\alpha}^{\alpha} \hat{\psi}(t) \mathrm{e}^{-\mathrm{i} t k_{n}}\left(1-\frac{\sigma^{2}}{2} L(t)\right)^{n} E\left(\mathrm{e}^{-\mathrm{i} t u}\right) E\left(\mathrm{e}^{-\mathrm{i} t v}\right) \mathrm{d} t \\
& =\frac{1}{2 \pi} \int_{-\alpha \sqrt{n}}^{\alpha \sqrt{n}} \hat{\psi}\left(\frac{t}{\sqrt{n}}\right) \mathrm{e}^{-\mathrm{i} t k_{n} / \sqrt{n}}\left(1-\frac{\sigma^{2}}{2} L\left(\frac{t}{\sqrt{n}}\right)\right)^{n} E\left(\mathrm{e}^{-\mathrm{i} \frac{t}{\sqrt{n}} u}\right) E\left(\mathrm{e}^{-\mathrm{i} \frac{t}{\sqrt{n}} v}\right) \mathrm{d} t \\
& \rightarrow \frac{1}{2 \pi} \int_{\mathbb{R}} \hat{\psi}(0) \mathrm{e}^{-\mathrm{i} t \kappa} \mathrm{e}^{-\frac{\sigma^{2}}{2} t^{2}} \mathrm{~d} t
\end{aligned}
$$

by dominated convergence. We have used the fact that $L(t) \sim t^{2}$ close to 0 , and in particular, if $\alpha$ is small enough, $\left(1-\frac{\sigma^{2}}{2} L(t / \sqrt{n})\right)^{n} \leqslant\left(1-\sigma^{2} t^{2} /(4 n)\right)^{n} \leqslant \mathrm{e}^{-\sigma^{2} t^{2} / 4}$, which gives the domination.

Set $\chi(\kappa)=\mathrm{e}^{-\kappa^{2} /\left(2 \sigma^{2}\right)} /(\sigma \sqrt{2 \pi})$. We have proved that, for any $\psi$ in $L^{1}$ with $\hat{\psi}$ compactly supported,

$$
\sqrt{n} E\left(\psi\left(S_{n} f-k_{n}-u-v \circ T^{n}\right)\right) \rightarrow \chi(\kappa) \int_{\mathbb{R}} \psi(x) \mathrm{d} x .
$$

Eq. (33) can then be extended to a larger class of functions by density arguments (see [7]), and this larger class contains in particular the characteristic functions of bounded intervals. This concludes the proof.

\subsection{The periodic case}

The following theorem gives the local limit theorem when the group $\mathfrak{A}$ of Paragraph 5.1 is a discrete subgroup of $\mathbb{R}$, for example $2 \pi \mathbb{Z}$.

Theorem 5.3 (local limit theorem, periodic case). Let $X$ be a Young tower with $\operatorname{gcd}\left(\varphi_{i}\right)=1$. Assume that $m[\varphi>n]=O\left(1 / n^{\beta}\right)$ with $\beta>2$. Let $\tau<1$. Take $f \in C_{\tau}(X)$ of zero integral, and $\sigma^{2}$ given by Theorem 1.1.

Assume that $f=\rho+q$ where $q$ takes integer values and $\rho \in \mathbb{R}$, but that $f$ cannot be written as $f=\rho^{\prime}+g-$ $g \circ T+\lambda q^{\prime}$, where $\lambda \in \mathbb{N}-\{1\}$ and $q^{\prime}: X \rightarrow \mathbb{Z}$ (this implies in particular $\sigma>0$ ). Then, for every sequence $k_{n}$ with $k_{n}-n \rho \in \mathbb{Z}$ such that $k_{n} / \sqrt{n} \rightarrow \kappa \in \mathbb{R}$,

$$
\sqrt{n} m\left\{x \in X \mid S_{n} f(x)=k_{n}\right\} \rightarrow \frac{\mathrm{e}^{-\kappa^{2} /\left(2 \sigma^{2}\right)}}{\sigma \sqrt{2 \pi}} .
$$

Proof. This is essentially the same proof as that of Theorem 1.2, but we use the Fourier transform on $\mathbb{Z}$ (i.e. Fourier series) instead of the Fourier transform on $\mathbb{R}$.

If $k$ and $l$ are two integer numbers,

$$
1_{k=l}=\frac{1}{2 \pi} \int_{-\pi}^{\pi} \mathrm{e}^{\mathrm{i} t(l-k)} \mathrm{d} t .
$$


Applying this equation to $k_{n}-n \rho$ and $S_{n} f(x)-n \rho$ and integrating gives

$$
m\left\{x \in X \mid S_{n} f(x)=k_{n}\right\}=\frac{1}{2 \pi} \int_{-\pi}^{\pi} \mathrm{e}^{-\mathrm{i} t k_{n}} E\left(\mathrm{e}^{\mathrm{i} t S_{n} f}\right) \mathrm{d} t .
$$

This is an analogue of (32). From this point on, the proof of Theorem 1.2 applies. The only problem is to check that, on $[-\pi,-\alpha] \cup[\alpha, \pi], I-R(z, t)$ is invertible for $z \in \overline{\mathbb{D}}$. This comes from the assumptions on $f$, which ensures that $I-R(z, t)$ is invertible as soon as $t \notin 2 \pi \mathbb{Z}$, by Proposition 5.1 and Corollary 5.2 , since $\mathfrak{A}=2 \pi \mathbb{Z}$.

\section{Proof of the central limit theorem with speed}

Proof of Theorem 1.3. The Berry-Esseen Theorem [12] implies that the result will be proved if we show that, for some $c>0$,

$$
\int_{-c \sqrt{n}}^{c \sqrt{n}} \frac{1}{|t|}\left|E\left(\mathrm{e}^{\mathrm{i}(t / \sqrt{n}) S_{n} f}\right)-\mathrm{e}^{-\left(\sigma^{2} / 2\right) t^{2}}\right| \mathrm{d} t=O\left(\frac{1}{n^{\delta / 2}}\right) .
$$

We first estimate the integral between $-1 / n$ and $1 / n$ :

$$
\begin{aligned}
\int_{-1 / n}^{1 / n} \frac{1}{|t|}\left|E\left(\mathrm{e}^{\mathrm{i}(t / \sqrt{n}) S_{n} f}\right)-\mathrm{e}^{-\left(\sigma^{2} / 2\right) t^{2}}\right| \mathrm{d} t & \leqslant \int_{-1 / n}^{1 / n} \frac{1}{|t|}\left|E\left(\mathrm{e}^{\mathrm{i}(t / \sqrt{n}) S_{n} f}\right)-1\right| \mathrm{d} t+\int_{-1 / n}^{1 / n} \frac{1}{|t|}\left|e^{-\left(\sigma^{2} / 2\right) t^{2}}-1\right| \mathrm{d} t \\
& \leqslant \int_{-1 / n}^{1 / n} \frac{1}{\sqrt{n}} E\left(\left|S_{n} f\right|\right) \mathrm{d} t+\int_{-1 / n}^{1 / n} \frac{\sigma^{2}}{2}|t| \mathrm{d} t .
\end{aligned}
$$

But $\int\left|S_{n} f\right| \leqslant n \int|f|$, whence we get $O(1 / \sqrt{n})$ for this term.

Let $L(t)$ be given by Proposition 4.5. Then, for small enough $c$,

$$
\begin{aligned}
& \int_{1 / n \leqslant|t| \leqslant c \sqrt{n}} \frac{1}{|t|}\left|E\left(\mathrm{e}^{\mathrm{i}(t / \sqrt{n}) S_{n} f}\right)-\mathrm{e}^{-\left(\sigma^{2} / 2\right) t^{2}}\right| \mathrm{d} t \\
& \leqslant \int_{1 / n \leqslant|t| \leqslant c \sqrt{n}} \frac{1}{|t|}\left|\left(1-\frac{\sigma^{2}}{2} L\left(\frac{t}{\sqrt{n}}\right)\right)^{n}-\mathrm{e}^{-\left(\sigma^{2} / 2\right) t^{2}}\right| \mathrm{d} t \\
& +\int_{1 / n \leqslant|t| \leqslant c \sqrt{n}} \frac{1}{|t|}\left|E\left(\mathrm{e}^{\mathrm{i}(t / \sqrt{n}) S_{n} f}\right)-\left(1-\frac{\sigma^{2}}{2} L\left(\frac{t}{\sqrt{n}}\right)\right)^{n}\right| \mathrm{d} t .
\end{aligned}
$$

Let us show that the second term satisfies

$$
\int_{1 / n \leqslant|t| \leqslant c \sqrt{n}} \frac{1}{|t|}\left|E\left(\mathrm{e}^{\mathrm{i}(t / \sqrt{n}) S_{n} f}\right)-\left(1-\frac{\sigma^{2}}{2} L\left(\frac{t}{\sqrt{n}}\right)\right)^{n}\right| \mathrm{d} t=O\left(\frac{1}{\sqrt{n}}\right) .
$$

Set $e(n, t)=C\left[1 / n^{\beta-1}+|t|\left(1 / n^{\beta-1}\right) \star\left(1-d t^{2}\right)^{n}\right]$. By Theorem 4.6,

$$
\left|E\left(\mathrm{e}^{\mathrm{i}(t / \sqrt{n}) S_{n} f}\right)-\left(1-\frac{\sigma^{2}}{2} L\left(\frac{t}{\sqrt{n}}\right)\right)^{n}\right| \leqslant e\left(n, \frac{t}{\sqrt{n}}\right) .
$$


Thus, it is enough to prove that

$$
\int_{1 / n \leqslant|t| \leqslant c \sqrt{n}} \frac{e(n, t / \sqrt{n})}{|t|} \mathrm{d} t=O\left(\frac{1}{\sqrt{n}}\right) .
$$

For the term $1 / n^{\beta-1}$ in $e(n, t)$, the integral is $C(\ln n) / n^{\beta-1}$, which is $O(1 / \sqrt{n})$.

For the other term $c(n, t)=|t|\left(1 / n^{\beta-1}\right) \star\left(1-d t^{2}\right)^{n}$, we cut the integral in two pieces. For $|t| \leqslant 1$, the convolution is bounded (since $1 / n^{\beta-1}$ is summable and $\left(1-d t^{2} / n\right)^{n} \leqslant 1$ ). It remains $\int_{1 / n}^{1} \frac{1}{|t|} \frac{|t|}{\sqrt{n}} \mathrm{~d} t \leqslant \frac{1}{\sqrt{n}}$. For $|t| \geqslant 1$, we use Lemma 3.2, which gives that $c(n, t) \leqslant 1 /\left(|t| n^{\beta-1}\right)+|t|\left(1-\frac{d}{2} t^{2}\right)^{n}$. Thus,

$$
\int_{1}^{\sqrt{n}} \frac{1}{|t|}\left|c\left(n, \frac{t}{\sqrt{n}}\right)\right| \mathrm{d} t \leqslant \int_{1}^{\sqrt{n}} \frac{\sqrt{n}}{t^{2} n^{\beta-1}} \mathrm{~d} t+\frac{1}{\sqrt{n}} \int_{1}^{\sqrt{n}} \mathrm{e}^{-d t^{2} / 2} \mathrm{~d} t=O\left(\frac{1}{\sqrt{n}}\right) .
$$

Finally, we have proved that

$$
\begin{aligned}
& \int_{-c \sqrt{n}}^{c \sqrt{n}} \frac{1}{|t|}\left|E\left(\mathrm{e}^{\mathrm{i}(t / \sqrt{n}) S_{n} f}\right)-\mathrm{e}^{-\left(\sigma^{2} / 2\right) t^{2}}\right| \mathrm{d} t \\
& \leqslant \int_{|t| \leqslant c \sqrt{n}} \frac{1}{|t|}\left|\left(1-\frac{\sigma^{2}}{2} L\left(\frac{t}{\sqrt{n}}\right)\right)^{n}-\mathrm{e}^{-\left(\sigma^{2} / 2\right) t^{2}}\right| \mathrm{d} t+O\left(\frac{1}{\sqrt{n}}\right) .
\end{aligned}
$$

We have only to deal with the powers of a function. Hence, it will be possible to use the same methods as in probability theory. More precisely, the study of the speed in the central limit theorem in [19, Theorem 3.4.1] uses the two following facts:

1. If a random variable $Z$ satisfies $E\left(|Z|^{2} 1_{|Z|>z}\right)=O\left(z^{-\delta}\right)$ with $0<\delta \leqslant 1$ and, in the $\delta=1$ case, $E\left(Z^{3} 1_{|Z| \leqslant z}\right)=$ $O(1)$, then there exists a constant $\lambda^{2} \geqslant 0$ such that $E\left(\mathrm{e}^{\mathrm{i} t Z}\right)=1-\frac{\lambda^{2}}{2} t^{2}(1+\gamma(t))$, with $\int_{-x}^{x} t^{2}|\gamma(t)| \mathrm{d} t=$ $O\left(x^{3+\delta}\right)$ when $x \rightarrow 0$.

2. If a function $\tilde{\gamma}(t)$ satisfies $\int_{-x}^{x} t^{2}|\tilde{\gamma}(t)| \mathrm{d} t=O\left(x^{3+\delta}\right)$ with $0<\delta \leqslant 1$, and $\sigma^{2} \geqslant 0$, then $\Lambda(t):=t^{2}(1+\tilde{\gamma}(t))$ satisfies $\int_{|t| \leqslant c \sqrt{n}} \frac{1}{|t|}\left|\left(1-\frac{\sigma^{2}}{2} \Lambda\left(\frac{t}{\sqrt{n}}\right)\right)^{n}-\mathrm{e}^{-\left(\sigma^{2} / 2\right) t^{2}}\right| \mathrm{d} t=O\left(n^{-\delta / 2}\right)$.

By Proposition 4.5, the eigenvalue $\lambda(t)$ of $\hat{T}_{B}(t)$ is equal to $E_{B}\left(\mathrm{e}^{\mathrm{i} t f_{B}}\right)+\alpha t^{2}+O\left(t^{3}\right)$ for some constant $\alpha$. The fact 1 applied to the random variable $f_{B}: B \rightarrow \mathbb{R}$ implies that $E_{B}\left(\mathrm{e}^{\mathrm{i} t f_{B}}\right)=1-\frac{\lambda^{2}}{2} t^{2}(1+\gamma(t))$ for some function $\gamma(t)$ satisfying $\int_{-x}^{x} t^{2}|\gamma(t)| \mathrm{d} t=O\left(x^{3+\delta}\right)$. As $\lambda(t)=1-\frac{\sigma^{2}}{2 m(B)} L(t)$, this implies that $L(t)=t^{2}\left(1+m(B) \frac{\lambda^{2}}{\sigma^{2}} \gamma(t)+\right.$ $O(t))$. Hence, we can write $L(t)=t^{2}(1+\tilde{\gamma}(t))$ with $\int_{-x}^{x} t^{2}|\tilde{\gamma}(t)| \mathrm{d} t=O\left(x^{3+\delta}\right)$. Therefore, the fact 2 implies that $\int_{|t| \leqslant c \sqrt{n}} \frac{1}{|t|}\left|\left(1-\frac{\sigma^{2}}{2} L\left(\frac{t}{\sqrt{n}}\right)\right)^{n}-\mathrm{e}^{-\left(\sigma^{2} / 2\right) t^{2}}\right| \mathrm{d} t=O\left(n^{-\delta / 2}\right)$.

By (34), we obtain $\int_{-c \sqrt{n}}^{c \sqrt{n}} \frac{1}{|t|}\left|E\left(\mathrm{e}^{\mathrm{i}(t / \sqrt{n}) S_{n} f}\right)-\mathrm{e}^{-\left(\sigma^{2} / 2\right) t^{2}}\right| \mathrm{d} t=O\left(n^{-\delta / 2}\right)$, which concludes the proof.

\section{Appendix. The Wiener Lemma}

In this appendix, we prove that the algebra $\mathcal{O}_{\gamma}(\mathcal{C})$ introduced in Paragraph 3.1 is indeed a Banach algebra, and that it satisfies a Wiener Lemma (Theorem A.3). 
Let $\mathcal{C}$ be a Banach algebra, and take $\gamma>1$. Write $w_{n}=(n+1)^{-\gamma}$ for $n \geqslant 0$. There exists a constant $c$ such that $\left(w_{n}\right) \star\left(w_{n}\right) \leqslant c w_{n}$. We define a norm on $\mathcal{O}_{\gamma}(\mathcal{C})$ by

$$
\left\|\sum_{n \in \mathbb{Z}} A_{n} z^{n}\right\|=\left(\sum_{n \in \mathbb{Z}}\left\|A_{n}\right\|+c \sup _{n \geqslant 0} \frac{\left\|A_{n}\right\|}{w_{n}}\right)+\left(\sum_{n \in \mathbb{Z}}\left\|A_{n}\right\|+c \sup _{n \leqslant 0} \frac{\left\|A_{n}\right\|}{w_{|n|}}\right) .
$$

Proposition A.1. Let $\mathcal{C}$ be a Banach algebra, and $\gamma>1$. With the norm (A.1), $\mathcal{O}_{\gamma}(\mathcal{C})$ is a Banach algebra.

Proof. The completeness is clear. It is sufficient to prove the submultiplicativity of the norm for one half of this norm, for example the first one. Let us write $\left\|\sum A_{n} z^{n}\right\|_{1}=\sum\left\|A_{n}\right\|$, and $P_{w}\left(\sum A_{n} z^{n}\right)=\sup _{n} \geqslant 0\left\|A_{n}\right\| / w_{n}$. Then, if $A=\sum A_{n} z^{n}$ and $B=\sum B_{n} z^{n}$, we have $\|A B\|_{1} \leqslant\|A\|_{1}\|B\|_{1}$. Moreover, for $n \geqslant 0$,

$$
\begin{aligned}
\frac{\left\|(A B)_{n}\right\|}{w_{n}} & \leqslant \frac{\sum_{k}\left\|A_{k} B_{n-k}\right\|}{w_{n}} \\
& \leqslant \frac{1}{w_{n}} \sum_{k=0}^{n}\left\|A_{k} B_{n-k}\right\|+\left(\sum_{k=-\infty}^{-1}\left\|A_{k}\right\| P_{w}(B) \frac{w_{n-k}}{w_{n}}\right)+\left(\sum_{k=-\infty}^{-1}\left\|B_{k}\right\| P_{w}(A) \frac{w_{n-k}}{w_{n}}\right) \\
& \leqslant P_{w}(A) P_{w}(B) \frac{(w \star w)_{n}}{w_{n}}+\|A\|_{1} P_{w}(B)+\|B\|_{1} P_{w}(A) .
\end{aligned}
$$

Thus, $P_{w}(A B) \leqslant c P_{w}(A) P_{w}(B)+\|A\|_{1} P_{w}(B)+\|B\|_{1} P_{w}(A)$. This gives the conclusion.

We will now identify the characters of the commutative algebra $\mathcal{O}_{\gamma}(\mathbb{C})$, i.e. the algebra morphisms from $\mathcal{O}_{\gamma}(\mathbb{C})$ to $\mathbb{C}$. For $\lambda \in S^{1}$, we can define a character $\chi_{\lambda}$ on $\mathcal{O}_{\gamma}(\mathbb{C})$ by $\chi_{\lambda}(a)=\sum a_{n} \lambda^{n}$.

Proposition A.2. The characters of $\mathcal{O}_{\gamma}(\mathbb{C})$ are exactly the $\chi_{\lambda}$, for $\lambda \in S^{1}$.

Proof. This result is given by Rogozin in [28], but there is a (density) problem in his argument, for $\gamma \notin \mathbb{N}$. A correction is given in [29], and a more direct argument can be found in [13, Theorem 1.2.12].

The following theorem has been thoroughly used in Section 3. It is a Wiener Lemma in the algebra $\mathcal{O}_{\gamma}(\mathbb{C})$.

Theorem A.3. Let $\mathcal{C}$ be a Banach algebra, $\gamma>1$, and $A(z)=\sum_{n \in \mathbb{Z}} A_{n} z^{n} \in \mathcal{O}_{\gamma}(\mathcal{C})$. Assume that, for every $z \in S^{1}$, $A(z)$ is an invertible element of $\mathcal{C}$. Then $A$ is invertible in the Banach algebra $\mathcal{O}_{\gamma}(\mathcal{C})$.

Proof. Gelfand's Theorem [31, Theorem 11.5 (c)] ensures that, if an element $a$ of a commutative Banach algebra satisfies $\chi(a) \neq 0$ for every character $\chi$, then $a$ is invertible. With Proposition A.2, this gives Theorem A.3 for $\mathcal{O}_{\gamma}(\mathbb{C})$.

To handle the case of a general noncommutative Banach algebra, we use Theorem 3 of [6].

The same kind of Wiener Lemma holds in the algebra $\mathcal{O}_{\gamma}^{+}(\mathcal{C})$ (also defined in Section 3.1):

Theorem A.4. Let $\mathcal{C}$ be a Banach algebra, $\gamma>1$, and $A(z)=\sum_{n \in \mathbb{N}} A_{n} z^{n} \in \mathcal{O}_{\gamma}^{+}(\mathcal{C})$. Assume that, for every $z \in \overline{\mathbb{D}}$, $A(z)$ is an invertible element of $\mathcal{C}$. Then $A$ is invertible in the Banach algebra $\mathcal{O}_{\gamma}^{+}(\mathcal{C})$.

Proof. This is the same proof as in Theorem A.3 (but here, the characters on $\mathcal{O}_{\gamma}^{+}(\mathcal{C})$ are given by $\chi_{\lambda}(a)=$ $\sum_{n=0}^{\infty} \lambda^{n} a_{n}$ for $\left.\lambda \in \overline{\mathbb{D}}\right)$. 


\section{References}

[1] J. Aaronson, An Introduction to Infinite Ergodic Theory, Mathematical Surveys and Monographs, vol. 50, American Mathematical Society, 1997.

[2] J. Aaronson, M. Denker, The Poincaré series of $\mathbb{C} \backslash \mathbb{Z}$, Ergodic Theory Dynam. Systems 19 (1999) 1-20.

[3] J. Aaronson, M. Denker, Local limit theorems for partial sums of stationary sequences generated by Gibbs-Markov maps, Stochastics and Dynamics 1 (2001) 193-237.

[4] J. Aaronson, B. Weiss, Remarks on the tightness of cocycles, Colloq. Math. 8485 (2000) 363-376.

[5] J.F. Alves, S. Luzzatto, V. Pinheiro, Markov structures and decay of correlations for non-uniformly expanding dynamical systems, Preprint, 2002.

[6] S. Bochner, R.S. Phillips, Absolutely convergent Fourier expansions for non-commutative normed rings, Ann. Math. 43 (1942) $409-418$.

[7] L. Breiman, Probability, Addison-Wesley, 1968.

[8] A. Broise, Transformations dilatantes de l'intervalle et théorèmes limites, Astérisque 238 (1996) 1-109.

[9] H. Bruin, S. Luzzatto, S. van Strien, Decay of correlations in one-dimensional dynamics, Ann. Sci. École Norm. Sup. 36 (2003) $621-646$.

[10] J.-P. Conze, S. Le Borgne, Méthode de martingales et flot géodésique sur une surface de courbure constante négative, Ergodic Theory Dynam. Systems 21 (2) (2001) 421-441.

[11] D. Dolgopyat, Limit theorems for partially hyperbolic systems, Trans. Amer. Math. Soc. 356 (2004) 1637-1689.

[12] W. Feller, An Introduction to Probability Theory and its Applications, vol. 2, Wiley Series in Probability and Mathematical Statistics, Wiley, 1966.

[13] J. Frenk, On Banach Algebras, Renewal Measures and Regenerative Processes, CWI Tract, vol. 38, Centrum voor Wiskunde en Informatica, Amsterdam, 1987.

[14] S. Gouëzel, Central limit theorem and stable laws for intermittent maps, Probab. Theory Related Fields 128 (2004) 82-122.

[15] S. Gouëzel, Regularity of coboundaries for non uniformly expanding Markov maps, Preprint, 2004.

[16] S. Gouëzel, Sharp polynomial bounds for the decay of correlations, Israel J. Math. 139 (2004) 29-65.

[17] Y. Guivarc'h, J. Hardy, Théorèmes limites pour une classe de chaînes de Markov et applications aux difféomorphismes d'Anosov, Ann. Inst. H. Poincaré Probab. Statist. 24 (1988) 73-98.

[18] H. Hennion, Sur un théorème spectral et son application aux noyaux lipschitziens, Proc. Amer. Math. Soc. 118 (1993) $627-634$.

[19] I.A. Ibragimov, Y.V. Linnik, Independent and Stationary Sequences of Random Variables, Wolters-Noordhoff, Groningen, 1971. With a supplementary chapter by I.A. Ibragimov and V.V. Petrov. Translation from the Russian edited by J.F.C. Kingman.

[20] C.T. Ionescu-Tulcea, G. Marinescu, Théorie ergodique pour des classes d'opérations non complètement continues, Ann. Math. 47 (1950) $140-147$.

[21] J. Komlós, A generalization of a problem of Steinhaus, Acta Math. Acad. Sci. Hungar. 18 (1967) 217-229.

[22] S. Le Borgne, F. Pène, Décorrélation multiple pour certains systèmes quasi-hyperboliques. Applications, Preprint.

[23] C. Liverani, Central limit theorems for deterministic systems, in: International Conference on Dynamical Systems, Montevideo 1995, in: Pitman Research Notes in Mathematics, vol. 362, 1996.

[24] C. Liverani, B. Saussol, S. Vaienti, A probabilistic approach to intermittency, Ergodic Theory Dynam. Systems 19 (1999) $671-685$.

[25] C.C. Moore, K. Schmidt, Coboundaries and homomorphisms for nonsingular actions and a problem of H. Helson, Proc. L.M.S. 40 (1980) $443-475$.

[26] M. Pollicott, R. Sharp, Invariance principles for interval maps with an indifferent fixed point, Comm. Math. Phys. 229 (2002) $337-346$.

[27] A. Raugi, Étude d'une transformation - non uniformément hyperbolique de l'intervalle [0, 1[, Bull. Soc. Math. France 132 (2004) 81-103.

[28] B. Rogozin, Asymptotic behavior of the coefficients in Levi-Wiener theorems on absolutely converging trigonometric series, Siberian Math. J. 14 (1973) 917-923.

[29] B. Rogozin, Asymptotic behavior of the coefficients of functions of power series and Fourier series, Siberian Math. J. 17 (1977) $492-498$.

[30] J. Rousseau-Egele, Un théorème de la limite locale pour une classe de transformations dilatantes et monotones par morceaux, Ann. Probab. 11 (1983) 772-788.

[31] W. Rudin, Functional Analysis, second ed., International Series in Pure and Applied Mathematics, McGraw-Hill, 1991.

[32] O. Sarig, Subexponential decay of correlations, Invent. Math. 150 (2002) 629-653.

[33] D. Szász, T. Varjú, Local limit theorem for Lorentz process and its recurrence in the plane, Ergodic Theory Dynam. Systems 24 (2004) 257-278.

[34] L.-S. Young, Statistical properties of dynamical systems with some hyperbolicity, Ann. of Math. (2) 147 (1998) 585-650.

[35] L.-S. Young, Recurrence times and rates of mixing, Israel J. Math. 110 (1999) 153-188.

[36] R. Zweimüller, Stable limits for probability preserving maps with indifferent fixed points, Stochastics and Dynamics 3 (2003) 83-99. 